\title{
Live-Structure Dataflow Analysis for Prolog
}

\author{
ANNE MULKERS \\ Katholieke Universiteit Leuven \\ and \\ WILLIAM WINSBOROUGH \\ Pennsylvania State University \\ and \\ MAURICE BRUYNOOGHE \\ Katholieke Universiteit Leuven
}

For the class of applicative programming languages, efficient methods for reclaiming the memory occupied by released data structures constitute an important aspect of current implementations. The present article addresses the problem of memory reuse for logic programs through program analysis rather than by run-time garbage collection. The aim is to derive run-time properties that can be used at compile time to specialize the target code for a program according to a given set of queries and to automatically introduce destructive assignments in a safe and transparent way so that fewer garbage cells are created.

The dataflow analysis is constructed as an application of abstract interpretation for logic programs. An abstract domain for describing structure-sharing and liveness properties is developed as are primitive operations that guarantee a sound and terminating global analysis. We explain our motivation for the design of the abstract domain, make explicit the underlying implementation assumptions, and discuss the precision of the results obtained by a prototype analyzer.

Categories and Subject Descriptors: D.3.4 [Programming Languages]: Processors-compilers; optimization; F.3.1 [Logies and Meanings of Programs]: Specifying and Verifying and Reasoning about Programs; I.2.3 [Artificial Intelligence]: Deduction and Theorem Proving-logic programming

General Terms: Languages, Performance

Additional Key Words and Phrases: Abstract interpretation, compile-time garbage collection, liveness, program analysis, Prolog

\section{INTRODUCTION}

In conventional languages, such as $\mathrm{C}$ or Pascal, the programmer explicitly controls the dynamic allocation of storage cells through declarations and destructive assignments. Applicative languages in their pure form do not

Anne Mulkers is supported by DPWB contract RFO/AI/02 (Belgium). William Winsborough is supported in part by the National Science Foundation (USA) grant CCR 88-05503. Maurice Bruynooghe is supported by the Belgian National Fund for Scientific Research.

Authors' addresses: A. Mulkers and M. Bruynooghe, Department of Computer Science, K. U. Leuven, Celestijnenlaan 200A, B-3001 Heverlee, Belgium; W. Winsborough, Department of Computer Science, Pennsylvania State University, University Park, PA 16802.

Permission to copy without fee all or part of this material is granted provided that the copies are not made or distributed for direct commercial advantage, the ACM copyright notice and the title of the publication and its date appear, and notice is given that copying is by permission of the Association for Computing Machinery. To copy otherwise, or to republish, requires a fee and/or specific permission.

(C) 1994 ACM 0164-0925/94/0300-0205\$3.50 
have destructive assignments. Also type declarations are often absent. Standard implementations of applicative programming languages keep the stored representation of a data structure intact when its parts are used to construct a new data structure, although there may be no subsequent reference to the old structure. A substantial overhead is incurred, since it is usually cheaper in time and memory usage to reuse the old value's storage. The run-time garbage-collecting processes that are called when the system runs out of memory deallocate the resulting dead data structures, but constitute a costly remedy, since they require an interruption of the program execution. Globalflow analysis techniques are being developed that are concerned with determining at compile time the type and scope of data structures that are dynamically created during program execution. The purpose of live-structure analysis is to derive at each program point which structures can have a subsequent reference during program execution. The compiler can exploit such structure liveness information to generate target code that reuses heap storage that is no longer referenced by program variables, i.e., to perform optimizations such as compile-time garbage collection [Bruynooghe et al. 1987], local reuse within a procedure [Foster 1989; Foster and Winsborough 1991], and, eventually, destructive update. All these optimizations try to reuse fields that already have the right value.

We address the problem of live-structure analysis for the class of pure Horn clause logic programs [Mulkers 1991; Mulkers et al. 1990a]. The language considered has a countable set of variables and countable sets of function and predicate symbols. A term is a variable, a constant, or a compound term $f\left(t_{1}, \ldots, t_{n}\right)$ where $f$ is an $n$-ary function symbol and the $t_{1}$ are terms. An atom has the form $p\left(t_{1}, \ldots, t_{m}\right)$ where $p$ is an $m$-ary predicate symbol and the $t_{t}$ are terms. A body is a (possibly empty) finite conjunction of atoms, written $A_{1}, \ldots, A_{k}$. A clause consists of an atom (its head) and a body and is written $A$ :- B. A program consists of a finite number of clauses. A query or goal consists of a body only, written ?-B. Features such as assert and retract are not considered, i.e., we assume that any source code for the predicates that can be executed at run time is available to the compiler.

We base our analysis on abstract interpretation [Cousot and Cousot 1977] of the source programs. A wide variety of abstract interpretation frameworks for logic programs has been constructed [Bruynooghe 1991; Debray 1992; Jones and Søndergaard 1987; Marriott and Søndergaard 1989; Mellish 1987]. Essentially, a framework is a parameterized construction of an analysis together with theorems that ensure the soundness (conservative upper approximation of the reachable states) and termination of the analysis constructed provided that the domains and operations supplied to complete the construction obey certain safety requirements. The task of constructing an analysis by using the framework is accomplished by supplying abstract domains and operations that obey the safety requirements, capture the information of interest, and, by properties of the domain, induce a terminating analysis.

The study of code optimization schemes that explicitly reclaim or reuse garbage cells is beyond the scope of the present article. Mariën et al. [1989]

ACM Transactions on Programming Languages and Systems, Vol. 16, No. 2, March 1994 
discussed some preliminary experiments on code optimization based on liveness information. Only opportunities for local reuse of storage cells are considered, i.e., reuse within the same clause where a cell is turned into garbage. Nonlocal reuse would require extra run-time data areas (e.g., a free list) to keep track of the location and size of free space. Although possible in principle, the standard heap allocation scheme would become significantly more complicated, and this overhead might overwhelm the performance gains. Therefore, nonlocal reuse of garbage cells is considered less beneficial for code optimization.

In Section 2, we identify the program properties that are of interest to derive structure liveness. In Section 3, we discuss the general method of abstract interpretation on which our global-flow analysis is based. In Section 4 , we define a variant of the concrete semantics capturing the information of interest and construct an appropriate abstraction. In Section 5, we illustrate the primitive operations and show that they indeed mimic standard implementations. In Section 6, we discuss the practical usefulness of liveness information in standard Prolog implementations. In Section 7, we comment on some possible alternative design options. In Section 8 , we discuss results obtained by a prototype implementation for a set of sample programs. In Section 9, we discuss related work in the context of functional and imperative programming languages. Sections $3.1,3.2$, and 5.4 provide some details on formal requirements and specifications useful for formally verifying the analysis; these sections can safely be skimmed by readers only interested in the basic ideas.

\section{THE ROLE OF STRUCTURE SHARING IN COMPUTING LIVENESS}

As an illustration of the use of live-structure information in Prolog programs, consider the program shown in Figure 1 for naive list reversal. If we assume that queries to nrev $/ 2$ are restricted to have as a first argument a list that is no longer referenced after the call, and as a second argument a free variable to return the output, then it is possible to generate for this program target code that allocates no new list constructor cells, but rather reuses the list cells of the first argument. Indeed, under the assumption, type analysis can infer that each call to the recursive clause of nrev/ 2 has as its first argument a list, and as a second argument a free variable. The unification of the call with the clause head selects the head and tail of the first argument list. The principal list constructor cell of the first argument has no subsequent references in the clause following the unification of the call with the clause head. This means that the compiler can recognize the principal list cell as garbage and generate target code that reuses it. For instance, consider the call to append $/ 3$ made by the same clause. A single element list $[E]$ needs to be constructed. Instead of allocating a new cell, the compiler can reuse the garbage cell that was detected.

In the above example, we can also infer that the first two arguments in each call to append / 3 will be lists and that the third argument will be a free variable. Again, it is possible to detect, that, after invocation of the recursive 


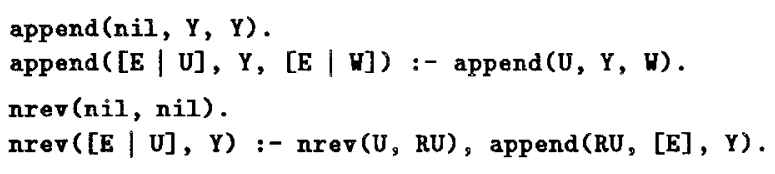

Fig. 1. Naive reverse.

clause of append $/ 3$, that the principal cell of the first argument is garbage and can be reused to construct the value of the third (output) argument. Thus, all list constructions in this example can reuse garbage list cells, eliminating all allocation operations. Since the reused cells would otherwise be garbage, we have eliminated the garbage collection overhead associated with the nrev $/ 2$ procedure. Moreover, a compiler can detect that the element field of each reused list cell already contains the value desired in the cell's new use. The operations filling in these car fields can be eliminated from the generated target code. The resulting code closely resembles the normal procedure in an imperative language for reversing a linear list of linked records.

However, the problem is more complex if there may be multiple references to the cells of the input list. Detecting the sharing of term substructure that can occur during program execution is a key prerequisite to derive liveness information. Because of such sharing, structure cells are not necessarily garbage immediately after their first inspection. Suppose the current resolvent has the form

$$
\leftarrow\left(\mathrm{A}_{i}, \mathrm{~A}_{i+1}, \ldots, \mathrm{A}_{q}\right) \sigma
$$

where $\left(\mathrm{A}_{l}, \ldots, \mathrm{A}_{q}\right) \sigma$ are the pending calls-either query calls or renamed copies of calls appearing in the bodies of the program clauses-and the substitution $\sigma$ is the current variable-binding environment. The left-to-right computation rule selects the call $\mathrm{A}_{\imath} \sigma$ for execution. Suppose the first argument of $A_{t}$ is $X$, and $\sigma$ binds $X$ to a structure, such as a list or a tree. Clearly, if $X$ also occurs in $A_{t+1}, \ldots, A_{q}$, then the structure to which $X$ is bound is live, and its storage must not be reused while executing $A_{1} \sigma$. However, this is not the only circumstance in which the structure is live. Most implementations of unification unify a variable and a structure by making the variable a reference to the structure-not a copy of the structure. In general, a cell in the structure to which $X$ is bound is live if any variable occurring in $A_{t+1}, \ldots, A_{q}$ is bound to a structure that contains the cell in question. Viewed in terms of the represented logical terms, liveness propagates through the sharing of structure in the terms' representations. Consequently, shared-structure analysis is a prerequisite of liveness analysis.

The sharing of structure is a property not of the standard semantics but of the language implementation. Our analysis is targeted to implementations that use the standard unification algorithm [Lassez et al. 1988] and perform all SLD derivations according to Prolog's left-to-right computation rule, which always resolves the leftmost goal in the current resolvent. They introduce 

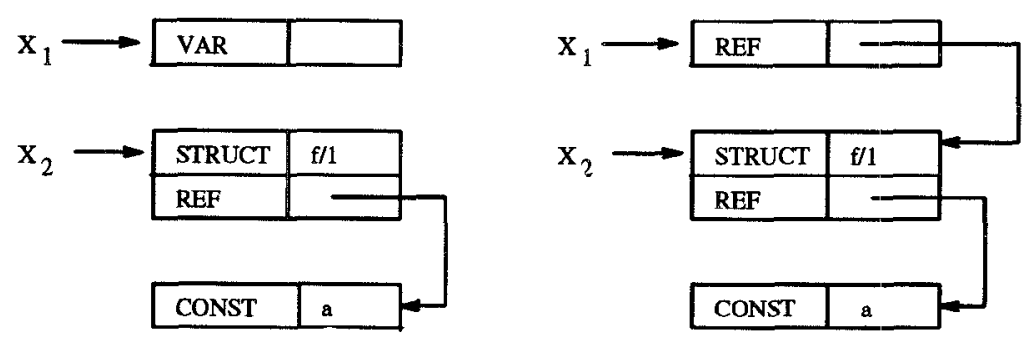

Fig. 2. Creation of structure sharing.

sharing of storage cells only to represent multiple occurrences of a variable (e.g., each occurrence eventually referring to a single storage location as in WAM-based implementations [Warren 1983] or all occurrences linked in a circular list of storage locations as in Taylor [1991]) and when binding a variable to a structure by loading the storage location representing the variable with a reference to the structure.

Figure 2 illustrates the effect of unification of two program variables $X_{1}$ and $X_{2}$ in a WAM-based implementation. In such implementations, execution environments representing the activated clauses are maintained on the so-called local stack. An environment contains information about the bindings of the clause variables and about how to proceed the execution. The heap or global stack contains the structures, lists, and variable cells created during program execution. A typical heap entry consists of a tag and a value cell. Possible tags are, for instance, REF (references), VAR (variables), STRUCT (structures), and CONST (constants). On the left in Figure 2, an initial variable-binding environment for the program variables is shown ( $\sigma_{i n}=\left\{X_{1} \leftarrow X_{1}, X_{2} \leftarrow f(a)\right\}$ ); the variable-binding environment after unifying both program variables is represented on the right $\left(\sigma_{\text {out }}=\left\{X_{1} \leftarrow f(a), X_{2}\right.\right.$ $\leftarrow f(a)\}$ ). Note that $X_{1}$ refers to the same storage cells as $X_{2}$. During program execution, the representations in memory of the logical terms to which variables are bound typically share some of their structure: while the denoted terms make up a forest of trees, their representations form a more general directed acyclic graph.

Our liveness analysis identifies substructures in the current call that are not currently referenced by any subsequent call in the current resolvent. When such structures are decomposed through unification of the call with a clause head, no live reference to the inspected upper cells is created. These cells are then candidates for reuse. Of course, care must be taken in the compiler to retrieve all needed values from such a cell before initializing it for another purpose.

Our liveness analysis considers only forward execution, ignoring backtracking. Because a scavenged structure may be needed again after backtracking, we assume some enhancement of the trailing mechanism of standard Prolog implementations. The trail records the write operations to memory cells that must be undone explicitly on backtracking. Our notion of liveness considers only forward execution of the subject program, i.e., a structure is live if it 
may be subsequently referenced without first backtracking past the current program point. The idea is that, when reusing a garbage cell, we replace the traditional WAM instruction put-structure, which allocates a cell on top of the heap, by use-old-structure, which takes the address of the cell to be reused as an argument. Before overwriting the cell, the new instruction performs a trail test and, if necessary, trails not only the address but also the content of the field to be overwritten, so that the cell can be completely restored on backtracking. Such an extension of the trail has also been proposed, for example, to assist implementation of delay mechanisms in logic programs [Boizumault 1988; Van Caneghem 1986] and for a scheme allowing the removal of dereferencing code in an optimizing compiler [Taylor 1990; 1991]. This value-trailing is more expensive than the WAM's address-trailing; in fact heap space is traded for space on the trail. However, the technique of destructive update will also avoid reinitialization of structure links that are already set correctly. As a consequence they need not be trailed, and a net gain in memory usage can be expected. Moreover, the trailing mechanism can be optimized when based on other simple dataflow analyses, e.g., Debray and Warren [1986b], Taylor [1989], and Van Roy and Despain [1990]. Nonetheless, it must be noted that in addition to determining the safety of reusing a given storage cell, the compiler should also do a cost benefit analysis to determine whether reuse is advisable. Such an analysis is beyond our current scope.

Note that the proposed method for local reuse of garbage cells does not interfere with the standard run-time garbage collection techniques. These will still be valid and useful because not all garbage cells created at run time will be detected by the liveness analysis.

Liveness analysis is traditionally formulated as a backward dataflow problem in which subsequent uses are propagated backward along control paths. On the other hand, the shared-structure analysis on which our liveness analysis is based is a forward dataflow problem. In order to allow us to perform these two analyses simultaneously, we make the following simplifying assumption: If a variable occurs in the current resolvent to the right of the first call, then the entire structure to which that variable is bound is considered live. Together with the absence of global references in logic programs, this assumption enables us to perform liveness analysis during a forward traversal of the program, simply by inspecting the remaining portion of each clause as control passes through it. This process will be clarified in Section 4.1.

\section{ABSTRACT INTERPRETATION OF LOGIC PROGRAMS}

Static program analysis is a general technique for deriving properties of the run-time behavior of a program. The information obtained in this way can be used to drive the optimization phase of a compiler or to guide source-level program transformation and program development tools. Often, program analysis can be viewed as execution of the program over a symbolic or abstract domain of data descriptions, instead of over the normal concrete data domain, and therefore it is called abstract interpretation. To mimic the 
concrete execution of a program, the basic operations defined on the standard domain are replaced by abstract operations defined on the abstract domain. The resulting flow analysis produces a so-called abstract semantics: for each reachable point of control in the program, it gives a finite description of the set of possible data states for the program when execution passes through that point. The program properties that compiler optimizations are based on are usually undecidable. Since static analyses are expected to be finitely computable, the data descriptions will be imprecise in general. The abstract interpretation is said to be sound if the data descriptions computed for each program point are conservative upper approximations of the set of concrete data states that can occur during program execution.

Cousot and Cousot [1977] provided a general framework for dataflow analysis problems of imperative languages, and they defined conditions which ensure the soundness of an abstraction. Based on that work, a variety of abstract interpretation frameworks has been developed for the specification and verification of analyses for logic programs [Bruynooghe 1991; Debray 1992; Jones and Søndergaard 1987; Marriott and Søndergaard 1989; Mellish 1987; Nilsson 1990]. A framework is a formally based, generic construction for program analysis that forms the basis for sound optimizations. To this end, it provides theorems that ensure the safety and termination of the analysis if the application-dependent domains and operations supplied to complete the construction obey certain safety requirements.

The standard theory of lattices provides the basic concepts for the technique of abstract interpretation. In order to apply the method for a new application, the space of properties (the abstract domain) capturing the information of interest should be a complete lattice (i.e., a set with a partial ordering such that each subset has a least upper bound and a greatest lower bound), and the functions used should be monotone or order-preserving on the lattice. If these conditions are satisfied, then the Tarski-Knaster Fixpoint Theorem [Tarski 1955] guarantees the existence of a solution for the fixpoint problem posed by the abstract semantics of the program.

The framework of abstract interpretation of Bruynooghe [1991] that forms the basis for our analysis is a so-called top-down analysis method and requires a set of similar but slightly weaker conditions. Top-down analyses are based on the standard operational semantics of Prolog, i.e., SLD resolution with a left-to-right computation rule. In order to derive the states in which clauses and their calls can be reached, a query specification is provided that characterizes the entry uses of the program. Data descriptions are then computed at a fixed number of program points: in each clause just after the head, between two calls in the body and after the last call.

The information inferred by the abstract interpretation process is gathered in an abstract AND-OR graph. Such a graph is the finite representation by means of a rational tree ${ }^{1}$ of a set of (possibly infinite) proof trees that

\footnotetext{
${ }^{1}$ A rational tree is a tree that has only a finite number of distinct subtrees (see A. Colmerauer, in Logic Programming, Academic Press, pp. 231-251).
} 
correspond to the concrete executions of the program. An Or-node represents a call, and its children represent the different clauses matching the call. The And-nodes on the other hand represent a clause head, and their children the subgoals of the clause body. Each program point is adorned with an element of the abstract domain, an abstract substitution. An initial abstract substitution representing a set of queries must be specified by the user. The other abstract substitutions computed by the abstract interpretation procedure for each program point will be safe approximations of the set of concrete substitutions that can actually occur at run time when control reaches such a point for any concrete derivation originating from one of the queries represented by the initial abstract substitution. The abstract substitution to the left of a call is often called the abstract call-substitution, and the one to the right is called the abstract success-substitution of the call.

Typical for the framework is that the abstract substitutions only mention the variables of the query or clause that they adorn. As a consequence, each call requires two unification-based operations, not just one as in standard SLD resolution. By confining attention to the state of the variables in one clause at a time, the analysis can recognize the similarity between clause invocations that differ only in the context of their use. This property is essential for the treatment of recursive calls. It also makes possible an optimization of the implementation such that a repeated computation of the same subgraph in different branches of the AND-OR graph is avoided. When a clause is invoked, the analysis restricts attention to the portion of the state pertaining to the variables in the clause. This restriction is achieved by an operation called procedure entry which abstracts the usual unification between call and clause head. When control returns to the caller, the analysis must reintroduce the variables in the calling environment and convey the effect of the clause execution upon those variables. This is achieved by an operation called procedure exit, which abstracts a second unification between call and (instantiated) clause head.

We introduce two further primitive operations into which we translate all nontrivial unification operations occurring in the subject program. A canonical form of the clauses is assumed: procedure calls and clause heads are normalized such that the parameters are distinct variables. This is done by breaking up unifications into sequences of simple operations. For example, the append $/ 3$ procedure in normal form is as follows.

append $(X, Y, Z):-X=$ nil, $Y=Z$.

$\operatorname{append}(X, Y, Z):-X=[E \mid U], Z=[E \mid W]$, append $(U, Y, W)$.

As a consequence parameter passing becomes very simple. It reduces to passing values from actual to formal parameters on procedure entry, and vice versa on procedure exit. The real unification work is done by the sequence of primitive unification operations. A unification call can have either of two forms: $X_{\iota}=X_{j}$ or $X_{t}=f\left(X_{l_{1}}, \ldots, X_{\iota_{j}}\right)$. If the language is extended with evaluable predicates (e.g., built-ins var $/ 1$, is $/ 2, \mathrm{arg} / 3, \ldots$ ), a primitive $a b$ stract operation has to be associated with each of them (see Cortesi and Filé [1991]). 
The safety requirements imposed by the framework (see Section 3.2) assure that the outcomes of abstract procedure entry, procedure exit, and primitive unification cover the outcomes of their concrete counterparts. Next, we first look at the requirements imposed on the abstract domain in order to get a finite analysis.

\subsection{Algebraic Structure of Concrete and Abstract Domains}

The set of variables in the clause (or query) that a concrete (or abstract) substitution adorns is called the domain $\mathrm{D}$ of the substitution. We denote the set of all idempotent concrete substitutions having domain $\mathrm{D}$ by ConcrSub $\mathrm{D}_{\mathrm{D}}$, and the set of all abstract substitutions having domain $D$ by AbstrSub ${ }_{D}$. The concretization function $\gamma$ defining the semantics of the abstract substitutions is a mapping

$$
\gamma: \text { AbstrSub }_{\mathrm{D}} \rightarrow 2^{\text {ConcrSub }_{\mathrm{D}}} .
$$

Although the framework does not refer explicitly to an abstraction function to yield the best overall description of a set of concrete substitutions,

$$
\alpha: 2^{\text {ConcrSub }_{\mathrm{D}}} \rightarrow \text { AbstrSub }_{\mathrm{D}},
$$

we assume such a function is given and allows the user to abstract the initial set of queries.

The set $A_{b s t r S u b}$ is not required to be finite, nor does it need to be a complete lattice; the abstract interpretation framework of Bruynooghe [1991] imposes a slightly weaker algebraic structure on $\mathrm{AbstrSub}_{\mathrm{D}}$ that still guarantees termination of the abstract interpretation procedure. There must exist a binary relation $\leq$ that is reflexive and transitive (a preordering) and that satisfies the following property

$$
\forall \beta_{1}, \beta_{2} \in \text { AbstrSub }_{\mathrm{D}}: \beta_{1} \leq \beta_{2} \Rightarrow \gamma\left(\beta_{1}\right) \subseteq \gamma\left(\beta_{2}\right) .
$$

We define an equivalence relation on the set $A_{b s t r S u b}$ as follows

$$
\forall \beta_{1}, \beta_{2} \in \text { AbstrSub }_{\mathrm{D}}: \beta_{1} \equiv \beta_{2} \Leftrightarrow \beta_{1} \leq \beta_{2} \& \beta_{2} \leq \beta_{1} .
$$

The relation $\leq$ can also be used modulo this equivalence relation, which yields a partial order for the set of equivalence classes. The existence of a uniquely determined representative for each equivalence class may be convenient, but is not required by the framework.

The framework further requires a maximal element $\beta_{\text {max }} \in$ AbstrSub $_{\mathrm{D}}$, a minimal element $\perp \in$ AbstrSub $_{\mathrm{D}}$, an upper-bound operator Upp: AbstrSub ${ }_{\mathrm{D}}$ $\times$ AbstrSub $_{D} \rightarrow$ AbstrSub $_{D}$, and a widening operator $\nabla:$ AbstrSub $_{D} \times$ AbstrSub $_{\mathrm{D}} \rightarrow$ AbstrSub $_{\mathrm{D}}$. A widening operator [Cousot 1981; Cousot and Cousot 1992] is a binary operator such that $\forall x, y \in \operatorname{AbstrSub}_{\mathrm{D}}:(x \leq x \nabla y) \&$ $(y \leq x \nabla y)$ and for all increasing sequences $x^{0} \leq x^{1} \leq \ldots$, the increasing sequence defined by $y^{0}=x^{0}, \ldots, y^{i+1}=y^{l} \nabla x^{i+1} \ldots$, is not strictly increasing. This operation is needed to enforce termination of the abstract interpre- 
tation procedure. The operation $\nabla$ is used instead of ( $R_{\circ}$ Upp) in previous work [Janssens 1990; Janssens and Bruynooghe 1992].

\subsection{Soundness Conditions for Primitive Operations}

We give the correctness conditions for the primitive abstract operations that guarantee soundness of the analysis. For details on the abstract interpretation procedure constructing the AND-OR graph we refer to Bruynooghe [1991]. The input for each primitive operation consists of a partially constructed AND-OR graph, a (collection of) abstract substitution(s), and a call represented by one of the nodes of the AND-OR graph. The output of the operation consists of a (set of) abstract substitution(s) and the updated AND-OR graph. We consider the AND-OR graph as a global data structure for which the updating is an implicit side-effect of the primitive operations. Below, the most general unifier of two terms $\mathscr{K}_{1}, \mathscr{K}_{2}$ is denoted by mgu( $\left.\mathscr{K}_{1}, \mathscr{K}_{2}\right)$. The restriction of a concrete substitution $\sigma$ to a set $\mathrm{D}$ of variables is denoted as $\left.\sigma\right|_{\mathrm{D}}$.

(1) Procedure-Entry $\left(\beta_{\imath n}, \mathrm{P}\right)=\left\{\beta_{\imath n}^{1}, \ldots, \beta_{\imath n}^{p}\right\}$

Applied to a call $\mathrm{P}$ with abstract call-substitution $\beta_{l n}$, the procedure-entry operation extends the abstract AND-OR graph at the Or-node representing the call $\mathrm{P}$, by adding a branch for each clause $\mathrm{H}^{j}:-\mathrm{B}_{1}^{J}, \ldots, \mathrm{B}_{m_{j}}^{J}$ $(1 \leq j \leq p$ ) defining the predicate $\mathrm{P}$ (see Figure 3 ), and by computing the abstract substitutions $\beta_{i n}^{1}, \ldots, \beta_{i n}^{p}$. The domain of the abstract substitution $\beta_{l n}^{J}$ is the set of variables $D_{j}$ in the $j$ th clause. The computation of the abstract substitutions is done in two steps out of convenience. First, the abstract substitution $\beta_{l n}$ is restricted by the call AbstrRestrict $\left(\beta_{l n}, \mathrm{P}\right)$ to a substitution $\beta_{l n}^{r s t r} \in A$ bstrSub ${ }_{D_{\mathrm{P}}}$, only referring to the domain $\mathrm{D}_{\mathrm{P}}$ of the call $P$. This step is independent of the set of clauses defining P. In the second step, $\beta_{l n}^{r s t r}$ is renamed for the domain of variables for each of the clauses, and the local variables of the clause are initialized.

The safety requirements that are sufficient for the correctness of the two steps of the procedure-entry operation are respectively:

(a) If $\sigma \in \gamma\left(\beta_{t n}\right)$ then $\left.\sigma\right|_{\mathrm{DP}} \in \gamma\left(\beta_{t n}^{r s t r}\right)$.

(b) For $1 \leq j \leq p$, if $\sigma \in \gamma\left(\beta_{\imath n}^{r s t r}\right)$ and $\theta=\operatorname{mgu}\left(\mathrm{P} \sigma, \mathrm{H}^{j}\right)$, with $\mathrm{H}^{j}$ the head of the properly renamed $j$ th clause defining $\mathrm{P}$, then $\left.\theta\right|_{D_{j}} \in \gamma\left(\beta_{i n}^{J}\right)$.

Due to the normal form of the source programs, proving the safety of the second step is rather straightforward for most applications. The first step may be more involved, especially for the application of liveness analysis.

(2) Procedure-Exit $\left(\beta_{\text {tn }},\left\{\beta_{\text {out }}^{1}, \ldots, \beta_{\text {out }}^{p}\right\}, \mathrm{P}\right)=\beta_{\text {out }}$

Procedure-exit is illustrated in Figure 4. It can be applied when the final abstract success-substitution $\beta_{\text {out }}^{J}$ has been derived for each clause defining $\mathrm{P}$. The initial step computes an abstract substitution $\beta_{\text {out }}^{\text {rstr }}$ that has as domain $D_{\mathrm{p}}$ the set of variables of the call $\mathrm{P}$. We have $\beta_{\text {out }}^{\text {rstr }}=\mathrm{Upp}$ $\left(\left\{\beta^{1}, \ldots, \beta^{p}\right\}\right)$, where $\beta^{J}(1 \leq j \leq p)$ is the renaming to $\mathrm{D}_{\mathrm{P}}$ of AbstrRestrict $\left(\beta_{\text {out }}^{J}, \mathrm{H}^{j}\right)$. In the second step, the extension operation AbstrExtend 


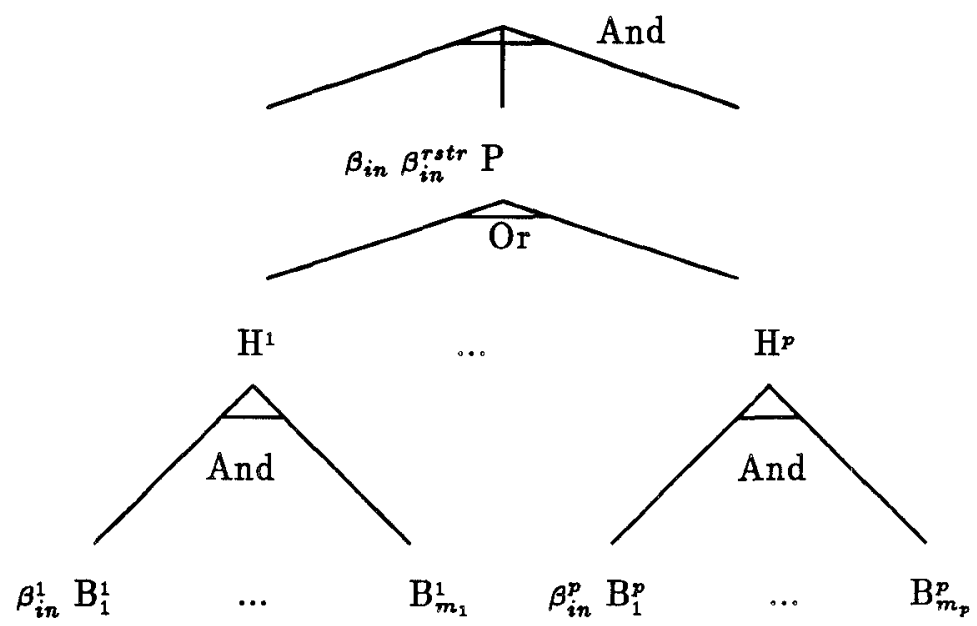

Fig. 3. Procedure-Entry extends the abstract AND-OR graph.

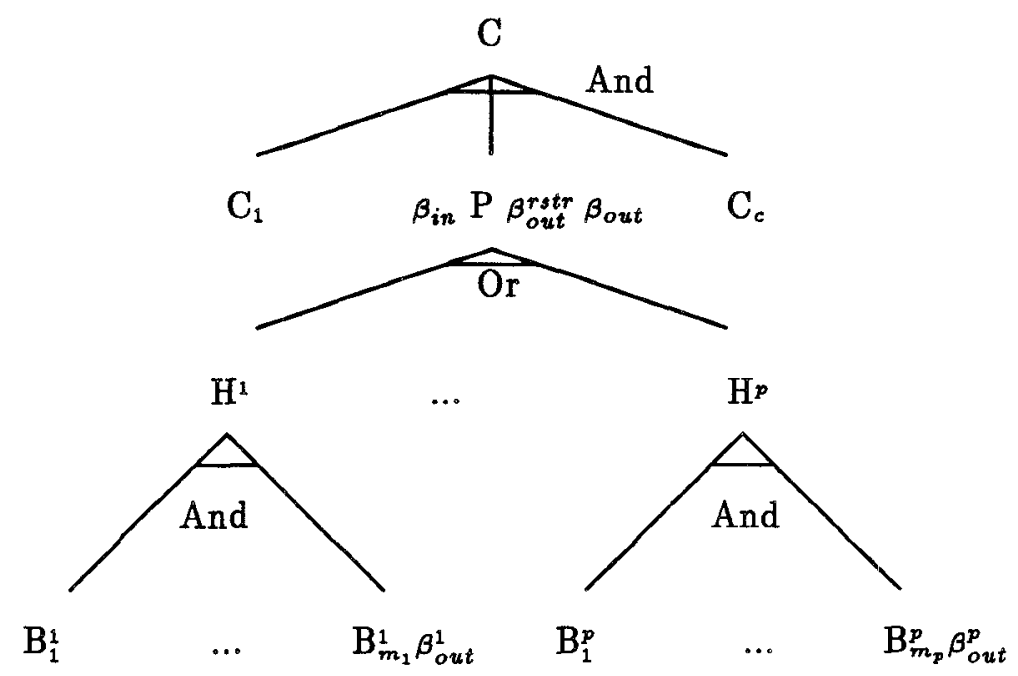

Fig. 4. Procedure-Exit computes $\beta_{o u t}$.

reintroduces the other variables in the domain $D_{\mathrm{Env}}$ of the calling environment (note: $\mathrm{D}_{\mathrm{P}} \subseteq \mathrm{D}_{\mathrm{Env}}$, where $\mathrm{D}_{\mathrm{Env}}$ is the domain of $\beta_{\imath n}$ and the calling clause $\mathrm{C}:-\mathrm{C}_{1}, \ldots, \mathrm{P}, \ldots, \mathrm{C}_{c}$ ), and makes explicit the effect on those variables of the execution of the procedure: $\beta_{\text {out }}=$ AbstrExtend( $\beta_{l n}$, $\left.\beta_{\text {out }}^{r \text { rstr }}, \mathrm{P}\right)$.

The safety requirements that are sufficient for the correctness of the two steps of the procedure-exit operation are respectively:

(a) If for some $j$ such that $1 \leq j \leq p, \sigma_{l n} \in \gamma\left(\beta_{l n}\right)$ and $\sigma_{J} \in \gamma\left(\beta_{o u t}^{J}\right)$, and there exists a substitution $\sigma$ such that $\mathrm{P} \sigma_{i n} \sigma=\mathrm{H}^{j} \sigma_{j}$, with $\mathrm{H}^{j}$ the head of the properly renamed $j$ th clause defining $\mathrm{P}$, and the only 
variables of $\left(\mathrm{C}:-\mathrm{C}_{1}, \ldots, \mathrm{P}, \ldots, \mathrm{C}_{c}\right) \sigma_{\imath n}$ occurring in $\sigma$ also occur in $\mathrm{P} \sigma_{l n}$, then $\left.\sigma_{i n} \sigma\right|_{\mathrm{D}_{\mathrm{P}}} \in \gamma\left(\beta_{\text {out }}^{r s t r}\right)$.

(b) If $\sigma_{\imath n} \in \gamma\left(\beta_{\imath n}\right)$ and there exists a substitution $\sigma$ such that $\left.\sigma_{\imath n} \sigma\right|_{\mathrm{D}_{\mathrm{P}}} \in$ $\gamma\left(\beta_{\text {out }}^{r s t r}\right)$, and the only variables of $\left(\mathrm{C}:-\mathrm{C}_{1}, \ldots, \mathrm{P}, \ldots, \mathrm{C}_{c}\right) \sigma_{i n}$ occurring in $\sigma$ also occur in $\mathrm{P}_{\sigma_{l n}}$, then $\left.\sigma_{\imath n} \sigma\right|_{\mathrm{D}_{\mathrm{E} n \mathrm{~V}}} \in \gamma\left(\beta_{\text {out }}\right)$.

The condition on the variables occurring in the substitution $\sigma$ says that $\sigma$ can be the result of executing $\mathrm{P} \sigma_{i n}$.

Proving the safety of the first step follows almost immediately from the properties of the Upp operation and the monotonicity of the concretization function $\gamma$.

(3) AbstrSimpleUnify $\left(\beta_{\imath n}, \mathrm{P}\right)=\beta_{\text {out }}$ (for $\left.\mathrm{P} \equiv X_{l}=X_{J}\right)$ AbstrFunctorUnify $\left(\beta_{l n}, \mathrm{P}\right)=\beta_{\text {out }}\left(\right.$ for $\mathrm{P} \equiv X_{\imath}=f\left(X_{i_{1}}, \ldots, X_{l_{j}}\right)$ )

Let $\mathrm{D}_{\mathrm{Env}}$ be the domain of $\beta_{\imath n}$. The safety requirement for the correctness of the abstract unification operations is: If $\sigma \in \gamma\left(\beta_{\imath n}\right)$ and executing $\mathrm{P} \sigma$ instantiates the call by $\theta$, then $\left.\sigma \theta\right|_{D_{\text {Env }}} \in \gamma\left(\beta_{\text {out }}\right)$.

\section{REPRESENTATION OF STRUCTURE SHARING AND LIVENESS}

From the discussion in Section 2, we know that an abstract domain for liveness analysis should provide a description of the structure of the values that can occur during program execution, the possible sharing of such structures, and the subset of live structures. Because we want to justify the abstract operations with respect to the concrete operations, we also need an instrumented concrete semantics that reflects the sharing and liveness of structures. These properties are normally not expressed by the standard semantics. So, we first introduce a variant of the standard concrete semantics that captures this information for the standard implementations of logicprogramming languages. Then we construct an approximating abstract semantics. The present section motivates the representations used in the instrumented concrete and abstract semantics. In Section 5 we present the operations on these domains.

\subsection{The Concrete Domain}

In order to formally verify our analysis for a class of implementations, it is important to model the sharing without modeling a particular memory organization. Standard implementations share structure among all occurrences of the same variable and introduce new shared structure when unifying two free variables or when unifying a free variable with a term. Rather than replacing the standard substitution environment with a representation that reveals memory references, we simply augment the variable-binding environment with information about which term occurrences in the environment can share their representations. Figure 5 illustrates the representations, called concrete sharing environments, for the variable-binding environments of Figure 2. Terms are represented as labeled trees. A variable-binding environment is represented as a tuple with the names of the program variables labeling the outarcs of the root node. Shared substructures are 

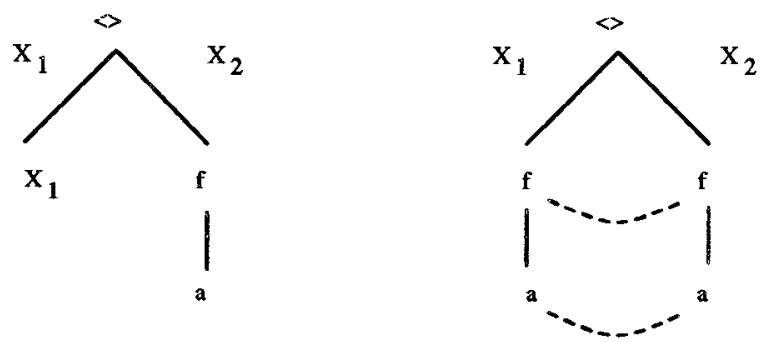

Fig. 5. Representation of the variable-binding environments of Figure 2.

shown as being connected by dashed arcs (undirected since the sharing relation is symmetric). Essentially we define an equivalence relation over the substructures in an environment, indicating which subterm occurrences are represented by the same storage cells. To avoid cluttering the figures, we do not show the reflexive arcs. Note that only identical subterms can share their representation, and corresponding substructures of shared structures also share. This means that sharing arcs may be shifted downward in a term. Often, we shall show only the principal sharing arcs.

The augmented concrete semantics represents the implementation aspects that are sufficient to reason about the sharing of structures. It omits the direction of references, the number and relative location of memory cells (whether on the local stack or on the heap), and the presence and length of reference chains. We do not want our analysis to impose any design decisions concerning those aspects of real implementations.

We call a concrete sharing environment wherein the live substructures are marked a concrete liveness environment. Naturally, the subset of marked nodes has to be closed with respect to the sharing relation, and substructures of live structures should also be live. Our figures show live substructures by marking the principal live nodes with a star.

As discussed in Section 2, we will consider as live the structure that a variable is bound to if the variable occurs after the first call in the current resolvent. We will also consider live any structure that shares with such a structure. This model of liveness ignores backtracking, as discussed in Section 2 . In order to facilitate passing the liveness information inductively down the proof trees, we introduce a distinction between globally and locally live structures. Figure 6 represents a partial proof tree when program point (P) is reached. Forward execution in pure Prolog is a depth-first left-to-right traversal of the proof tree. Triangles denote completed subtrees. The current resolvent is

$$
\leftarrow\left(\mathrm{B}_{J}, \mathrm{~B}_{J+1}, \ldots, \mathrm{B}_{m}, \mathrm{D}_{k}, \ldots, \mathrm{D}_{q}, \ldots, \mathrm{A}_{i+1}, \ldots, \mathrm{A}_{n}\right) \sigma,
$$

with $\sigma$ the current variable-binding environment. The left-to-right computation rule selects the call $\mathrm{B}_{j} \sigma$ for execution. The path of active calls, as represented by the run-time control structures of an interpreter, consists of the calls $Q, A_{i}, \ldots, P, B_{J}$. Under a forward execution regime, term structures 
Fig. 6. A partial proof tree.
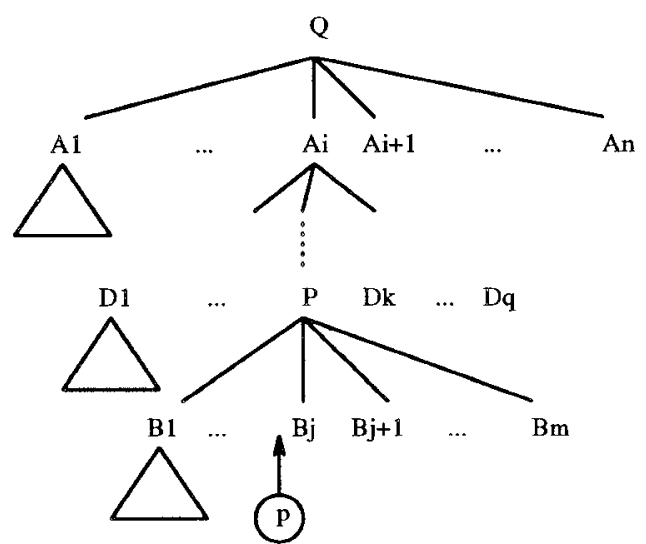

that represent $\mathrm{B}_{j} \sigma$ and that are shared with the term structures of $\left(\mathrm{B}_{j+1}, \ldots, \mathrm{A}_{n}\right)_{\sigma}$, must not be reused by the code generated for the clause that resolves with $\mathrm{B}_{j} \sigma$; such structures must be marked as live structures, because they will probably be referenced in a later stage of the execution. We call a structure globally live if it may be referenced in a subsequent program point in one of the ancestor clause instances. For Figure 6, the structures that $\mathrm{B}_{j} \sigma$ shares with $\left(\mathrm{D}_{k}, \ldots, \mathrm{D}_{q}, \ldots, \mathrm{A}_{t+1}, \ldots, \mathrm{A}_{n}\right) \sigma$ are globally live. A structure is called locally live, if it may be referenced in one of the subsequent program points of the current clause instance. For Figure 6, these are the structures that $\mathrm{B}_{j} \sigma$ shares with $\left(\mathrm{B}_{j+1}, \ldots, \mathrm{B}_{m}\right)_{\sigma}$. The distinction is convenient when processing the liveness information, because the set of locally live structures depends on the program variables referenced in the tail of the current clause, while the set of globally live structures does not. Global and local liveness for logic programs correspond to inter- and intraprocedural liveness for imperative programs. In the figures illustrating concrete liveness environments, nodes decorated with ${ }^{*}$ are locally live; nodes decorated with ** are globally live. A structure that is both locally and globally live will be simply decorated with ${ }^{* *}$ in the figures.

As discussed in Section 2, liveness analysis requires a kind of backward reasoning. The set of locally live terms is determined by the remainder of the clause body after the current program point. For the initial query, the set of live terms is initialized as the set of desired output structures. However, because the liveness depends on the structure-sharing information, which is obtained by a forward analysis, the computation of the liveness information is organized in a top-down, forward way.

\subsection{The Abstract Domain}

The type system of Bruynooghe and Janssens [1988], Bruynooghe et al. [1987], Janssens [1990], and Janssens and Bruynooghe [1992] forms the basis of our abstract domain to represent sharing and liveness of term structures. It introduces the concept of type graphs, which are essentially top-down deterministic tree automata [Gècseg and Steinby 1984]. They describe sets of 

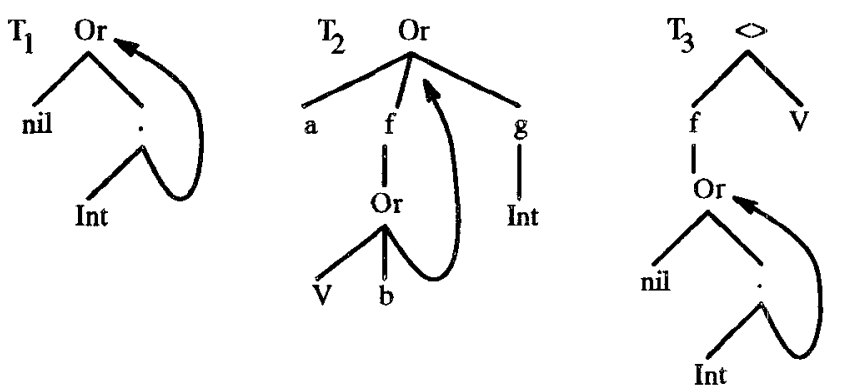

Fig. 7. The graphical representation of some type graphs.

partially instantiated terms, as well as ground terms, and provide the structure information necessary to express sharing and liveness of subterms at an adequate level of granularity.

We introduce type graphs only at an intuitive level. A type graph $\mathscr{T}=$ $\left\langle\right.$ Nodes $_{\bar{F}}$, ForwardArcs ${ }_{\mathscr{F}}$, BackArcs $\left._{\bar{Y}}\right\rangle$ is defined as a tree $\mathscr{T}_{\text {tree }}=\left\langle\right.$ Nodes $_{\mathscr{T}}$, ForwardArcs $\left.\bar{S}_{\overline{5}}\right\rangle$ supplemented with a (possibly empty) set of directed back-arcs connecting a node to one of its proper ancestors with respect to $\mathscr{T}_{\text {tree }}$. Each node of $\mathscr{T}$ is labeled by a function symbol, "Or," "V," $\perp$, or a primitive type name (Int, Real, ...). Each node labeled with a function synmbol has ordered children corresponding to the function symbol's arguments. ${ }^{2}$ Each node labeled Or has two or more unordered children. Other nodes have no children. The label of a node $n \in$ Nodes $_{\mathscr{T}}$ is denoted Label $(n)$. The root of the tree $\mathscr{T}_{\text {tree }}$ (and of the type graph $\mathscr{T}$ ) is denoted $\operatorname{Root}(\mathscr{T})$.

Figure 7 gives the graphical representation of some type graphs. In the figure, the nodes of a type graph are represented by their label. We do not explicitly indicate the direction of tree edges. We use the convention that the tree edges are drawn downward, while back-arcs are drawn upward. The root of the type graph is the topmost node.

The information described by a type graph can also be expressed by a context-free grammar. We use the convention that (nonterminal or primitive) type symbols start with a capital letter and that the other functor node labels consist of small letters only. For example, the type graphs represented in Figure 7 can be defined as:

$$
\begin{aligned}
& \mathrm{T}_{1}::=\text { nill.'. }\left(\text { Int, } \mathrm{T}_{1}\right), \\
& \mathrm{T}_{2}::=\mathrm{alf}\left(\mathrm{V}|\mathrm{b}| \mathrm{T}_{2}\right) \mid \mathrm{g}(\mathrm{Int}), \\
& \mathrm{T}_{3}::=\left\langle f\left(\mathrm{~T}_{1}\right), \mathrm{V}\right\rangle .
\end{aligned}
$$

The concretization TGConc( $\mathscr{T}$ ) of a type graph $\mathscr{T}$ is the set of concrete terms that it represents. It is the set of terms recognized by the type graph considered as a finite automaton or the language accepted by the context-free

\footnotetext{
${ }^{2} \mathrm{~A}$ unique natural number $n$ is associated with each function symbol $f$ and referred to as the arity of the symbol. A function symbol of arity 0 is referred to as a constant. The outdegree of a node labeled with a function symbol is equal to the arity of the function symbol.
} 
grammar that can be derived from the type graph. Here, it is understood that the nodes labeled Int represent the set of integers, the nodes labeled Real the set of real numbers. The node labeled $\perp$ represents the empty set of terms. The node labeled V represents the set of all variables.

Example. For the type graphs of Figure 7, we have

$$
\begin{aligned}
& \text { TGConc }\left(\mathrm{T}_{1}\right)=\{\text { nil, }[1,2,3],[4,4], \ldots\}, \\
& \text { TGConc }\left(\mathrm{T}_{2}\right)=\{a, f(X), f(b), f(a), f(f(a)), f(f(Y)), g(7), f(g(5)), \ldots\}, \\
& \text { TGConc }\left(\mathrm{T}_{3}\right)=\{\langle f([1,2,3]), X\rangle,\langle f(n i l), Y\rangle, \ldots\} .
\end{aligned}
$$

In Bruynooghe and Janssens [1988], Janssens [1990], and Janssens and Bruynooghe [1992] a number of restrictions on the form of type graphs are introduced. Some restrictions merely eliminate redundant nodes, while others reduce the number of sets expressible by type graphs. The restricted class of type graphs is better behaved than the unrestricted class, while still highly expressive. We make use of one restriction in particular. We assume that all type graphs satisfy the principal label restriction: Let

$$
\begin{aligned}
\operatorname{PrincipalNodes}(n)= & \text { if } \operatorname{Label}(n) \neq{ }^{\prime} O r^{\prime} \text { then }\{n\} \text { else } \\
& \cup\{\operatorname{PrincipalNodes}(m) \mid m \text { is a child of } n\},
\end{aligned}
$$

then for all nodes, $n$, labeled Or,

$$
m_{1}, m_{2} \in \operatorname{PrincipalNodes}(n): m_{1} \neq m_{2} \Rightarrow \operatorname{Label}\left(m_{1}\right) \neq \operatorname{Label}\left(m_{2}\right) .
$$

The principal label restriction makes the type graph deterministic as a recognizer of terms.

In the examples, we will also refer to the depth restriction of type graphs. For every function symbol, the depth restriction indicates the maximum number of nodes on a path in $\mathscr{T}_{\text {tree }}$ starting from the root node that can have the same function symbol as label. The restriction is used in the definition of a widening operator for the domain of type-graph environments needed to enforce termination of the abstract interpretation procedure. The depth bound will also turn out to be an important parameter to tune the precision of the sharing and liveness analysis. The particular value of the bound can be fixed for the whole program, or chosen on a per clause and functor basis according to some heuristic.

For the purposes of a correct and precise type inference system, a type graph is augmented with information about what positions in the denoted terms can be occupied by the same variable. The type graph $\mathscr{T}$ captures the structure of the terms; the sharing of free variables within those terms is captured by the variable-sharing component. We call a type graph $\mathscr{T}$ augmented with a variable-sharing component an abstract term environment and denote it as $\mathscr{T}^{\text {e }}$. We do not discuss variable sharing since for our application it serves only to strengthen the type graph analysis results. We regard $\mathscr{T}^{e}$ as an abstract data type that hides the variable-sharing component but exports the type graph $\mathscr{T}$.

In the present work, we assume that all type graphs have as their root a node that is not labeled Or. Each type graph used to represent a set of 

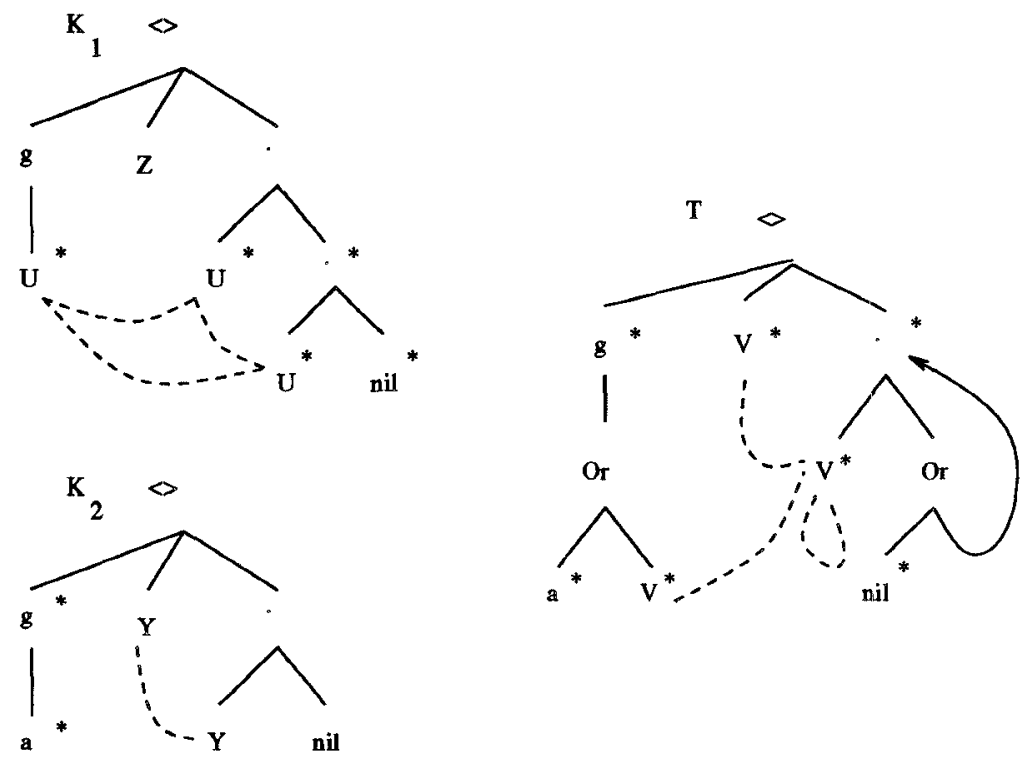

Fig. 8. Concrete and abstract liveness environments.

variable-binding environments will have its root labeled by either the tupling functor, \langle\rangle , with arity corresponding to the number of program variables in the current environment or $\perp$. We augment the type graphs by adding sharing and liveness components by analogy with the concrete liveness environments. An abstract sharing component for a type graph $\mathscr{T}$ represents the sharing that is possible in the terms represented by the type graph $\mathscr{T}$. The sharing is represented by means of dashed arcs connecting nodes having the same functor label. In Figure 8, an example of an abstract liveness environment is shown on the right and two of the concrete liveness environments it represents on the left. Note that for a term $\mathscr{K}$ represented by a type graph $\mathscr{T}$, a mapping can be defined associating each node of $\mathscr{K}$ with one node of $\mathscr{T}$ labeled with the same functor. Free variables in a concrete term match variable nodes (labeled V) in the type graph; multiple occurrences of the same variable must match type graph variable nodes that are connected by a sharing arc; live concrete nodes must match live type graph nodes (but not vice versa because the abstract liveness environments only represent possible liveness). Note that because of back-arcs, a node in a type graph can correspond to several nodes in a concrete term represented by the type graph. As a consequence, abstract reflexive arcs can be significant in the case of recursive types (i.e., type graphs with back-arcs) and represent nonreflexive concrete sharing arcs. While the concrete sharing relation is an equivalence relation, we do not require this property in the abstract case, because the abstract domain would not be fine enough. For instance, it would not be possible to distinguish between cases where the abstract reflexive arcs can correspond to nonreflexive concrete sharing arcs in a concrete execution state 
and cases where they cannot. As will become clear in the examples of the next section, the ability to make this distinction is crucial for the precision of the liveness analysis in order to detect opportunities for compile-time garbage collection. Therefore, only the significant reflexive arcs are included in the abstract sharing component, and the abstract sharing relation is required to be symmetric but not transitive.

\section{OPERATIONS COMPUTING STRUCTURE SHARING AND LIVENESS}

We will now discuss the primitive operations of unification, procedure entry, and procedure exit for the domains of concrete and abstract liveness environments. They compute the sharing and liveness of structured terms since they occur in standard implementations of logic-programming languages.

\subsection{Concrete Instrumented Unification and the Underlying Implementation Assumptions}

We first consider the operation SimpleUnify corresponding to the basic operation $X_{l}=X_{j}$, mimicking the effect of unifying two program variables in the presence of sharing and liveness information. The operation will be formalized by Definition 5.12 in Section 5.4.2. The other basic unification operation FunctorUnify, corresponding to $X_{\imath}=f\left(X_{\iota_{1}}, \ldots, X_{\imath \jmath}\right)$, requires only a minor extension [Mulkers 1991; Mulkers et al. 1993].

Let $\sigma_{l n}$ be the substitution represented by the variable-binding environment of Figure 9(a), prior to the unification of program variables $X_{1}$ and $X_{2}$.

$$
\sigma_{\imath n}=\left\{X_{1} \leftarrow h(X, f(Z)), X_{2} \leftarrow h(g(Y), Y)\right\}
$$

The resulting variable-binding environment is shown in Figure 9(b). For the term part, the effect of unification is expressed by applying the most general unifier of $X_{1} \sigma_{\iota n}$ and $X_{2} \sigma_{\imath n},\{X \leftarrow g(f(Z)), Y \leftarrow f(Z)\}$ (defined for example as in Lassez et al. [1988]). For the sharing part, the effect of unification is expressed by means of additional arcs indicating positions where unification has introduced new sharing of subterms. The language implementations that we address create sharing when binding a variable to an existing structure or another variable. Each variable occurrence in the input terms that are to be unified is determined, and an arc is introduced connecting the same position in the output environment and the corresponding position in the other term being unified. In this way, the instrumented unification operation simulates the creation of a reference in a real implementation. Because most Prolog implementations do not create any sharing when a variable gets bound to an atom or an integer, a small optimization of the SimpleUnify operation consists in avoiding the introduction of such sharing arcs. The new arcs are then shifted down according to the instantiation of the output environment to express that subterms of shared structures also share. Similarly, the sharing that existed in the input term prior to the unification is preserved and extended downward if the term is further instantiated by the unification. Finally, because the new sharing propagates through the old sharing for the representations of terms that we consider, the transitive closure operation is 


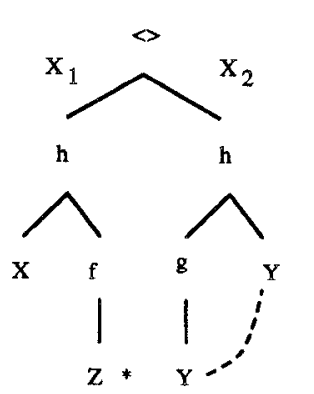

(a)

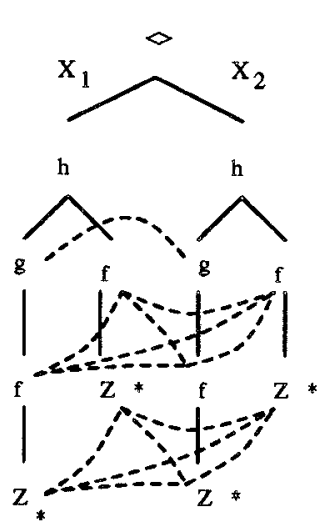

(b)

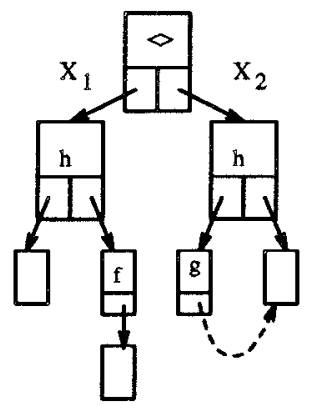

(c)

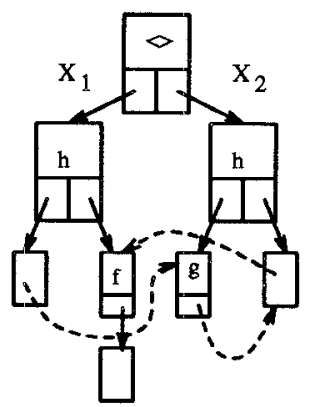

(d)

Fig. 9. Concrete input (a) and output (b) liveness environments for the SimpleUnify operation, the DAGs in a WAM-based implementation before (c) and after (d) unification.

applied making all sharing arcs explicit. The computation of the liveness component involves the application of a similar closure operation to propagate the liveness across new sharing arcs.

Standard structure-copying implementations, like WAM, create only sharing captured by the SimpleUnify operation. Terms are represented as directed acyclic graphs (DAGs) in memory. See Figures 9(c)-(d) for an illustration. The cell representing the root of the environment can be thought of as the frame on the local stack representing the current clause's execution environment, while the other cells are located on the heap. A sharing arc between two nodes of a variable-binding environment expresses that they are represented by the same dereferenced storage cell in the DAG. This is a variable cell, the address of a record on the heap, or the address of a constant. As discussed previously, the properties of the memory model require the sharing relation to be an equivalence relation closed with respect to the shift operation. Another characteristic property of WAM-based implementations is that they use a single variable cell to represent all occurrences of the same variable. The unification algorithm typically traverses the subterms to be unified synchronously until it hits a variable cell and then inserts a pointer from the variable cell to the other element (which can be a variable or a structure). From that point on, the dereferenced variable refers to the same storage cell as the other element. Taylor [1991] describes a different implementation and an optimizing compiler aimed at minimizing dereferencing code. To that end, multiple occurrences of the same variable are represented by a circular list of storage cells. When the variable is unified with a structure, a pointer to the structure is inserted in each of the storage cells of the circular list representing the aliases of the variable. If the variable is unified with another variable, the variable cell is inserted in the circular list. For both implementations, the creation of references is reflected in the 

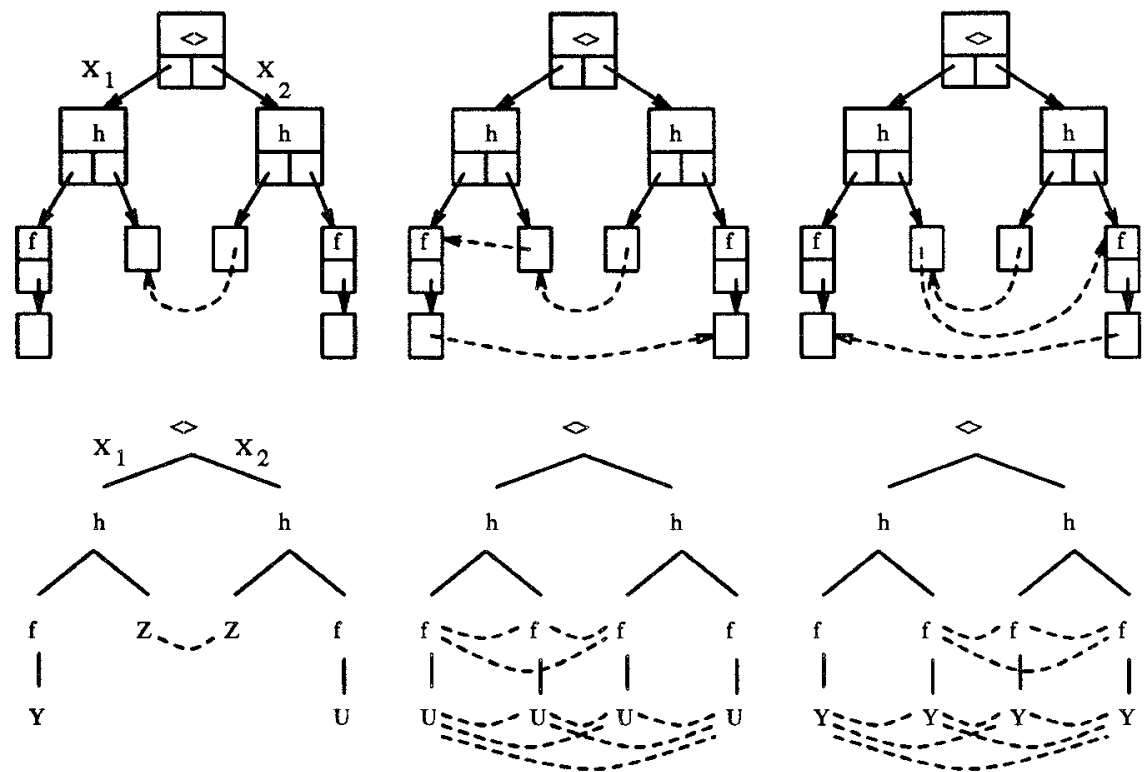

Fig. 10. The order in which unification subproblems are solved affects the sharing created.

instrumented unification algorithm by adding new arcs to the concrete sharing component.

The order in which unification subproblems are solved affects the sharing created. An illustration is given in Figure 10. In the top figures, a possible memory layout is sketched for a WAM-based implementation. On the left, a binding state is shown prior to the unification of two program variables $X_{1}$ and $X_{2}$. Depending on whether the arguments of corresponding functors are unified in the order from left-to-right or vice versa, we end up with different representations as is shown in the middle and right top figures respectively. In the bottom figures, the corresponding concrete sharing environments are shown. The concrete instrumented unification operation captures the sharing that can be introduced by unifying such dynamic structures in any order. The set of sharing arcs derived for this example is the transitively closed union of the sets of arcs shown in the bottom middle and right figures. In fact the SimpleUnify operation yields an upper approximation of the sharing created in any particular implementation. We expect the redundancy introduced by this strategy to be neglectable. In any case, mimicking the order used in one particular implementation at the abstract level of the operation would require a much closer integration between the type graph unification and the structure-sharing analysis, an option we did not consider because we think the increased precision will not be worth the extra complexity.

The example above also illustrates how different unification is from the functional cons, car, $c d r$, and the imperative new and assignment operations. Because of the invertibility property of logic programs, there is no simple translation from Prolog programs to Lisp or Pascal programs. Data 


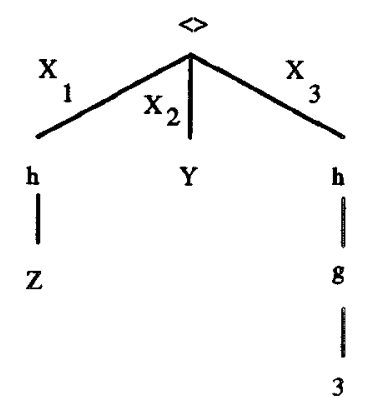

(a)

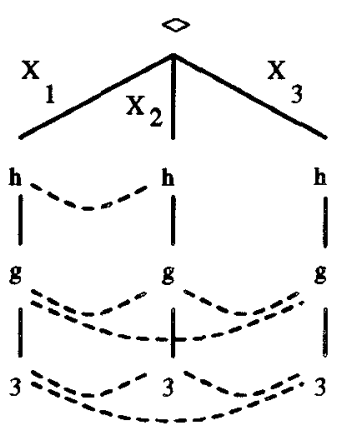

(b)

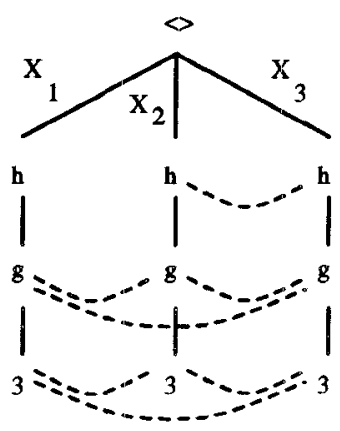

(a)

Fig. 11. The normal form chosen affects the sharing created.

manipulation in Prolog (record allocation as well as record access and parameter passing) is achieved entirely via unification. The sharing introduced by this general operation is more complicated to analyze at compile time than traditional parameter passing, term construction, and subterm selection; whether and where new references are introduced upon unification depends on the run-time values being unified, e.g., if the initial variable-binding environment consisted of ground terms only, no sharing would be created at all.

Note that the order in which static substructures are unified is predetermined by the normal form of the program. For instance, the program fact $p\left(X_{1}, X_{1}, X_{1}\right)$ could be transformed to the normal-form clauses $p\left(X_{1}, X_{2}, X_{3}\right):-X_{1}=X_{2}, X_{2}=X_{3}$, or $p\left(X_{1}, X_{2}, X_{3}\right):-X_{2}=X_{3}, X_{1}=X_{2}$. In Figure 11(a) we show a possible initial sharing environment; Figures 11(b)-(c) illustrate the concrete sharing environments at the end of the two normal-form clauses respectively. We assume that the normal form used reflects the program transformations (e.g., reordering of argument unifications) as performed by the optimization algorithm of the underlying compiler.

\subsection{Example Execution of a Program}

Apart from unification, the operations required to complete the parameterized abstract interpretation framework in order to induce a particular analysis are procedure entry and procedure exit. In this section, we first discuss the concrete version of each operation, using an example, and then we consider its abstract counterpart. The formal constructions and verifications of these operations are presented in Mulkers [1991] and Mulkers et al. [1993].

The first operation is procedure entry. To illustrate the issues involved we refer to Figure 12, which shows the naive list reversal program in normal form. In the figures, clause variables are renamed apart (to avoid variable capture) in each clause invocation by adding a subscript corresponding to that invocation. We first consider the program point marked $P_{1}$ just prior to the recursive call to nrev/2. Suppose that the environment involves the variable instances $X_{1}, Y_{1}, E_{1}, U_{1}, R U_{1}$, and Last ${ }_{1}$ and that when control reaches 


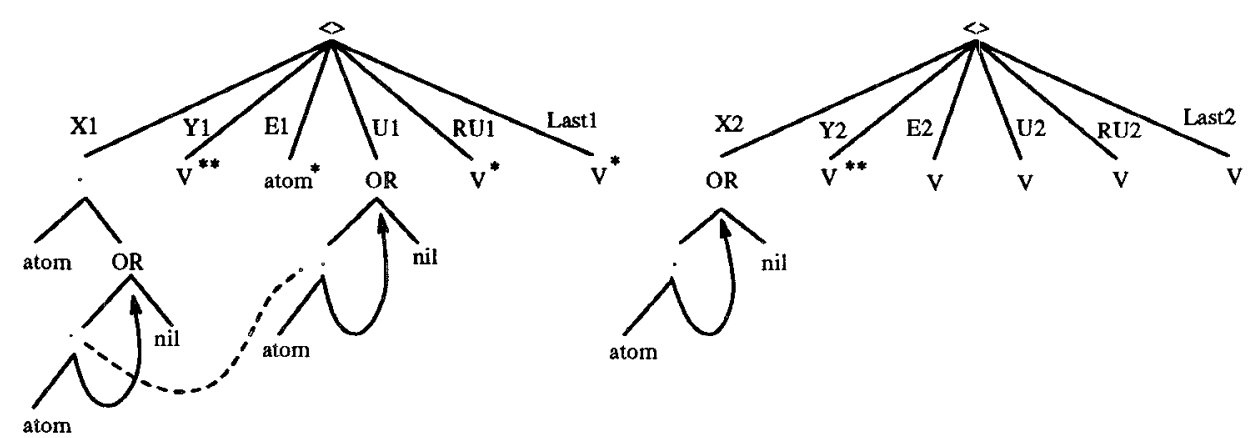

Fig. 14. Abstract liveness environment at point $P_{1}$ and then at point $P_{0}$ in the recursive invocation.

(labeled ${ }^{* *}$ ). In our example, this process gives the concrete liveness environment shown on the right in Figure 13.

At the abstract level, although terms are replaced by type graphs, the process is similar. Nodes of type graphs needed to complete the computation are determined; the information is restricted to the type graphs for $U_{1}$ and $\mathrm{RU}_{1}$, the arguments of the call, and passed on to $\mathrm{X}_{2}$ and $\mathrm{Y}_{2}$, the arguments of the head. Figure 14 illustrates the abstract analogue of the operation shown in Figure 13. Note that due to back-arcs, the principal sharing arcs for a type graph may be shifted upward in order to derive all implied sharing arcs.

The concrete unification of two variables of a liveness environment is discussed in the previous section. At the abstract level, the effect of unification is first to modify the type graphs, say $\mathscr{T}$ into $\mathscr{T}$. As in the concrete case, structures that are (possibly) sharing before unification remain (possibly) sharing after unification. To that end, we have to reexpress the sharing component for $\mathscr{T}$ as a component for $\mathscr{T}$. This is straightforward if we can determine corresponding nodes in both these type graphs. As type graphs are essentially deterministic, top-down tree automata, the problem is very similar to the well-known solved problem of determining whether two automata recognize intersecting sets. Next, abstract unification introduces new sharing where a variable is unified against some other structure. These new sharing arcs are collected and mapped onto the output type graph $\mathscr{T}$. When $\mathscr{T}$ has live nodes, updating the liveness component entails determining the corresponding nodes in the new type graph $\mathscr{T}$ and propagating liveness down new descendent arcs and across new sharing arcs.

Finally, we consider procedure exit. Figure 15 shows a concrete liveness environment in an invocation of nrev at the point the final append is called (program point $P_{2}$ ). Figure 16 shows the concrete liveness environment of the append clause at the point execution of the body is completed. The restriction of this state to the variables $X_{2}, Y_{2}$, and $Z_{2}$ has to be passed back to $R U_{1}$, Last $_{1}$, and $Y_{1}$, respectively. No liveness information need be returned. The result is shown in Figure 17. We see that $Y_{1}$, a live variable, has been bound to the list $[\mathrm{b}, \mathrm{a}]$, and that due to the generated sharing Last ${ }_{1}$ has become live, as well. 


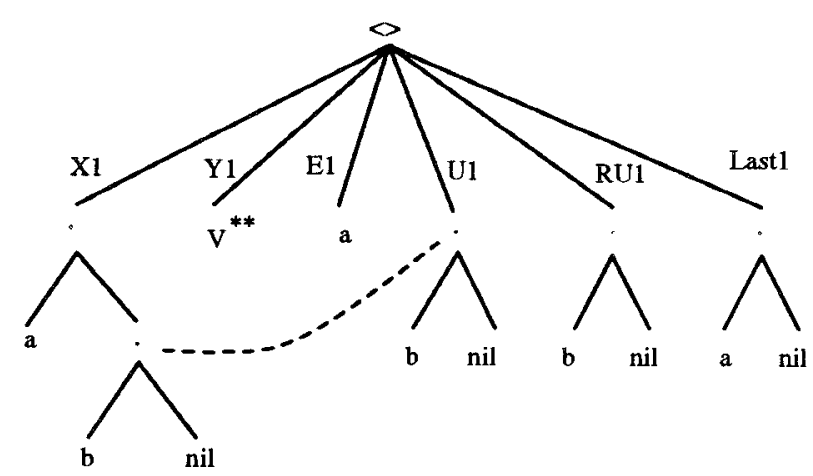

Fig. 15. The concrete liveness environment in the caller's environment at point $P_{2}$ - first of two inputs to procedure exit.

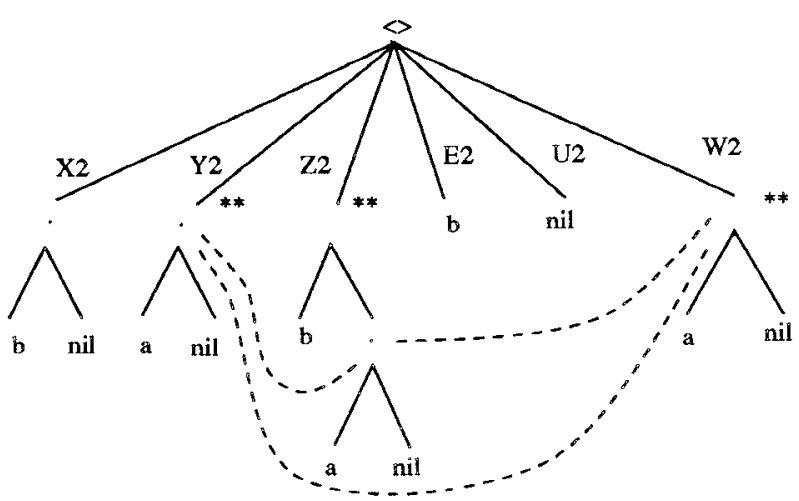

Fig. 16. The concrete liveness environment in the callee's environment at point $P_{4}$-second of two inputs to procedure exit.

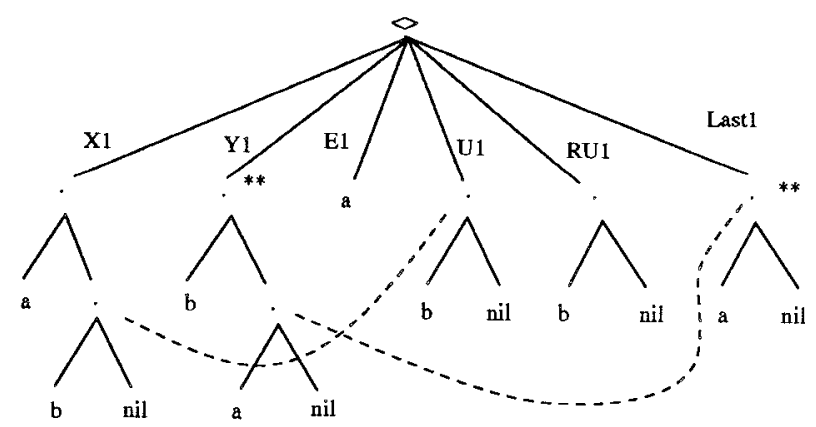

Fig. 17. Concrete liveness environment in caller's environment at point $P_{3}$-output for procedure exit.

ACM Transactions on Programming Languages and Systems, Vol. 16, No. 2, March 1994 


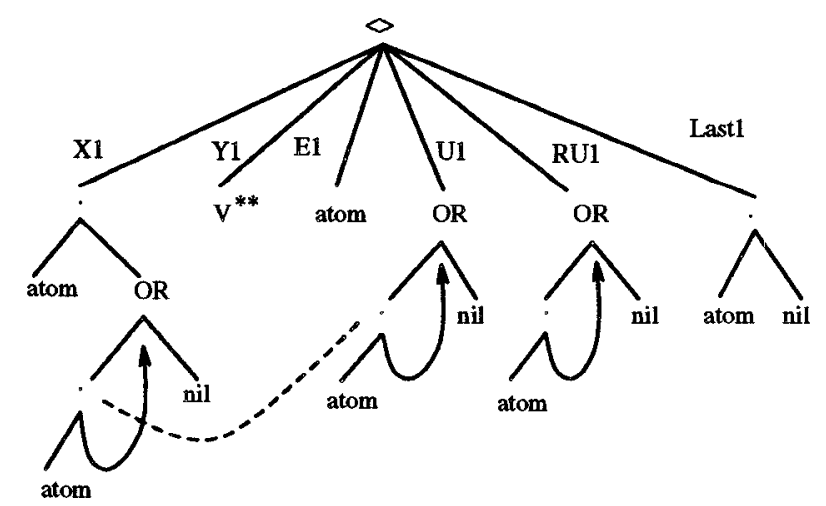

Fig. 18. The abstract liveness environment in the caller's environment at point $P_{2}$-first of two inputs to procedure exit.

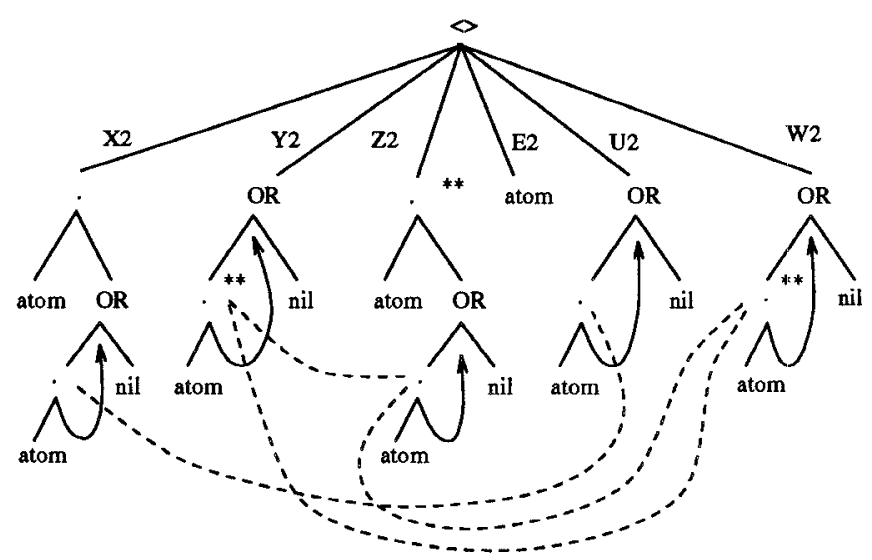

Fig. 19. The abstract liveness environment in the callee's environment at point $P_{4}$ - second of two inputs to procedure exit.

When moving from the concrete to the abstract level, we have similar, although more complex, operations. The abstract liveness environment must be restricted to the variables occurring in the head of the clause, and the type graphs and sharing information has to be returned to the caller. In a rather complex operation (called extension), these updates of the variables participating in the call have to be propagated among all the variables in the caller's environment: Further type graphs may need to be modified owing to shared variables; sharing has to be updated in terms of the new type graphs; the new sharing interacts with the sharing present in the caller's environment before the call, inducing further sharing. Also, the liveness component in the caller's environment has to be updated to take into account the modifications in the type graphs and the extension of the sharing. Figures 18-20 illustrate the 


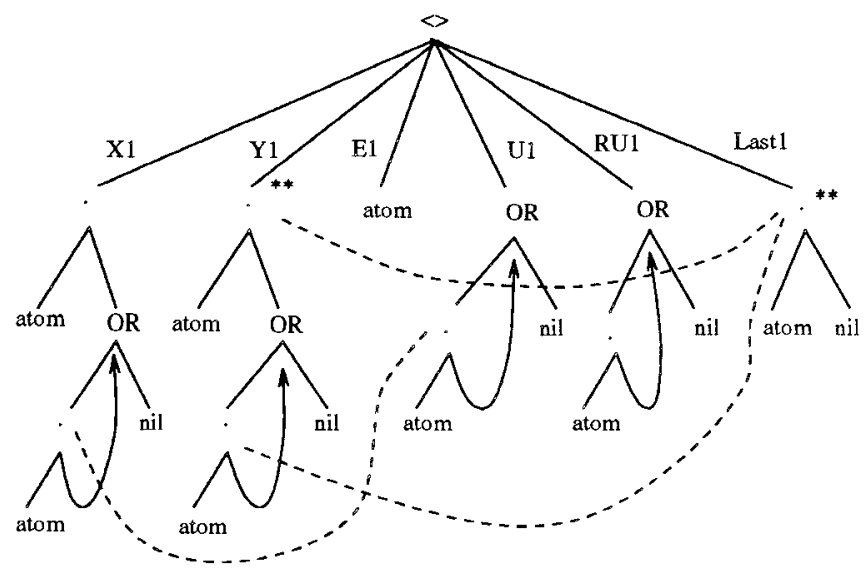

Fig. 20. Abstract liveness environment in caller's environment at point $P_{3}-$ output for procedure exit.

abstract analogue of the operation shown in Figures 15-17. Notice the possible sharing between $Y_{1}$ and Last ${ }_{1}$ in Figure 20, which is not present in Figure 17. This is due to the possibility that $\mathrm{RU}_{1}$ is the empty list in Figure 18.

\subsection{Abstract Operations}

In this section, we discuss in more detail some of the differences between the concrete and abstract operations. Recall that in the case of concrete liveness environments, we used the transitivity property for the sharing relation and the transitive closure operation to mimic the propagation of sharing. One of the main consequences of abstracting concrete terms and their definite sharing arcs, by means of type graphs and corresponding possible sharing arcs, is that the transitivity property is no longer appropriate. It introduces reflexive arcs that would make the abstract domain inappropriate for the application of liveness analysis. By dropping the transitivity requirement and using an alternating-closure operation, we obtain a level of precision that is satisfactory for several data-manipulating programs.

The idea of alternating paths is an adaptation of a similar idea proposed by Plaisted [1984] to detect points in Prolog programs where the occur check might be violated. Later it was also used by Søndergaard [1986] in a flow analysis to detect shared variables. Unlike transitive closure, alternating closure adds only arcs between nodes connected by a sequence of arcs in which the (old) sharing arcs of the input type graphs and the (new) arcs created by an operation on those graphs (e.g., unification) alternate.

Consider the first recursive clause of the program in Figure 21 for inserting an (integer) element into a sorted binary tree. Figure 22 shows the abstract sharing environments before and after the unification operation $O T=t(L, F, R)$ for the case where there is no internal sharing in the input binary tree passed 


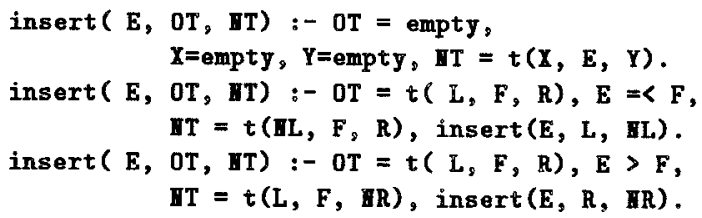

Fig. 21. insert $/ 3$.

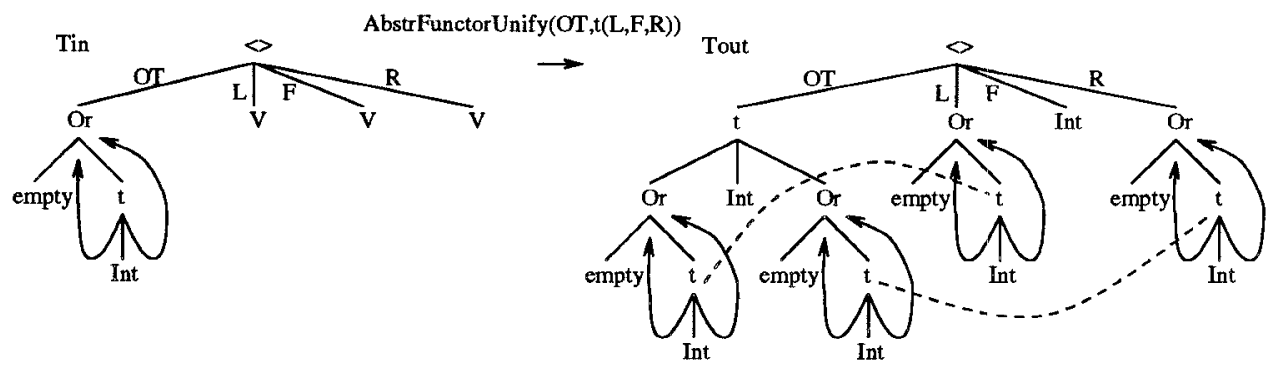

Fig. 22. Abstract unification: $\mathrm{OT}=\mathrm{t}(\mathrm{L}, \mathrm{F}, \mathrm{R})$.

to OT and that alternating closure is used. $^{3}$ If the abstract unification operation was based on the transitive closure operation, the new arcs resulting from the unification would entail reflexive arcs in the output type graph at the functor nodes labeled $t$ of both the subgraphs for the program variables $L$ and $R$. So, the recursive call insert(E, $L, N L)$ would have an input binary tree passed to $L$ for which possible internal sharing between its subtrees is assumed; hence the opportunity for in-place reuse of tree cells would not be recognized for the recursive call.

Moreover, by examining the trace of an abstract computation, we found that using twofold sharing components, preserving the distinction between old (input) and new (created) sharing arcs at the current clause level, improves the precision of the abstract extension operation. The full set of sharing arcs, consisting of the alternating closure of these two components, can be left implicit most of the time. Consider the recursive clause of the append $/ 3$ program, called with the first two arguments linear lists of integers that possibly share at the list cell level and the third argument a free variable (Figure 23(a)).

$$
\operatorname{append}(X, Y, Z):-X=[E \mid U], Z=[E \mid W], \operatorname{append}(U, Y, W)
$$

At the program point preceding the recursive call, we have the abstract sharing environment shown in Figure 23(b). The ares (1-3) and (2-3) are old

${ }^{3}$ Depth bound two is used for the tree constructor nodes.

ACM Transactions on Programming Languages and Systems, Vol. 16, No. 2, March 1994. 


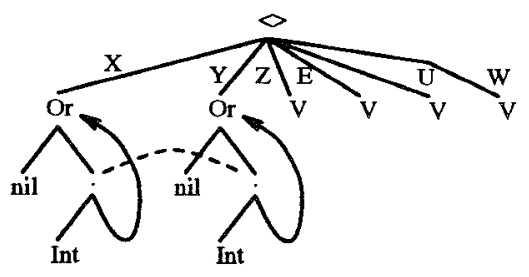

(a)

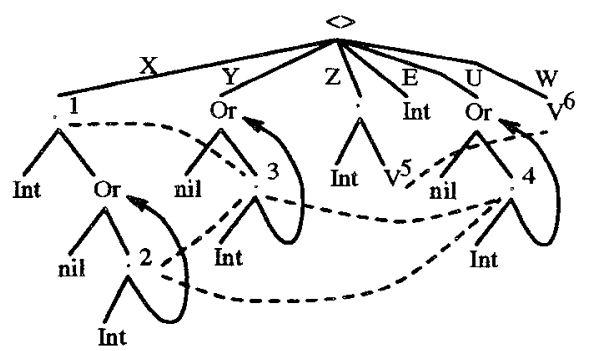

(b)

Fig. 23. Abstract sharing environments for the append/3 program, at the entry point (a) and before the recursive call (b).

arcs (resulting from the sharing environment at the entry point of the clause). The arcs (2-4) and (5-6) are new arcs resulting from the unifications $X=[E \mid U], Z=[E \mid W]$ in the current clause invocation. The arc (3-4) follows from the alternating closure. If the distinction is not maintained until clause exit, all the edges will be passed up as output edges. The extension operation to be applied after the call, will then have a difficult task in deciding whether the sharing edges that are both input and output represent possibly new sharing created by the call. For instance, for the recursive call append $(U, Y, W)$, the sharing between the list cells of $U$ and $Y$ will be both input and output. Clearly, a safe and easy extension operation exists for an abstract domain of sharing environments that contain only a single sharing component. This extension operation considers all output-sharing arcs of a call as possibly created by the call. However, for the example at hand, such an operation would yield an imprecise result, as it would consider the sequence of arcs $(2-3-4-2)$ as an alternating sequence. A self-edge in the list node ".2" would be generated, indicating that the input list $X$ possibly ends up with internal sharing of structure after the recursive call.

Of course, one can argue that, in this particular case where the input terms are ground, no new sharing would be introduced in the input list $X$ by the implementations that we consider. Instead of treating sharing components conservatively as binary relations in sets of nodes, we could define the primitive operations in such a way that they take into account the concretization of the subgraphs. By comparing the input and the output type graphs, we can decide whether sharing arcs involving ground parts of the output type graphs can be introduced as a result of executing the operation. This would resolve the imprecision introduced in the example above. In the general case of partially instantiated structures however, the initial sharing and the newly accumulated sharing cannot be distinguished from one another by merely comparing the input and output type graphs. The decomposition of the sharing component in two parts is better suited to solve the problem in all cases and improve the accuracy of the live-structure analysis. In fact, it is also more efficient because it yields a more compact representation of the sharing environments, and on procedure exit only the new sharing has to be 
passed upward in the AND-OR graph (the old sharing is already available in the calling environment).

\subsection{Formal Specifications}

After informally introducing the primitive operations in Section 5.1 and 5.2 and motivating the use of twofold sharing components in Section 5.3, we are now in the position to present the formal specifications.

5.4.1 Concrete and Abstract Domain. We begin by introducing some auxiliary notions that allow us to discuss the sharing and liveness of structure in the representation of terms. For a fixed enumeration of program variables, $X_{1}, \ldots, X_{c}$, a concrete term environment $\mathscr{K}$ is a term $\left\langle\mathscr{K}_{1}, \ldots, \mathscr{K}_{c}\right\rangle$, which represents the substitution $\left\{X_{1} \leftarrow \mathscr{K}_{1}, \ldots, X_{c} \leftarrow \mathscr{K}_{c}\right\}$. In this way, we have a canonical description of the substitution that is independent of variable naming. Following Huet [1980], an occurrence in a term $\mathscr{K}$ is a finite sequence of integers describing a position in $\mathscr{K}$. The indicated position is arrived at by following a path from the root, selecting each arc in the path according to the successive integers of the occurrence. We use ordered pairs of occurrences to indicate the positions of subterms whose representations are shared, and single occurrences to indicate live subterms. In our notation, $\epsilon$ denotes the empty sequence; "." denotes the operation of sequence concatenation; Vars denotes the (infinite) set of variables that can occur in terms; $\operatorname{Vars}(\mathscr{K})$ denotes the set of variables that occur in a particular term $\mathscr{K}$; and $\equiv$ denotes syntactic identity.

Definition 5.1. For a (possibly nonground) term $\mathscr{K}$, the set of occurrences in $\mathscr{K}, \mathscr{O}(\mathscr{K})$, is defined as follows.

$$
\mathscr{O}(\mathscr{K})=\{\epsilon\} \cup\left\{i . s \mid \begin{array}{l}
\mathscr{K} \equiv f\left(\mathscr{K}_{1}, \ldots, \mathscr{H}_{c}\right), \text { for some functor } f \\
\text { of arityc },(1 \leq i \leq c), \text { and } s \in \mathscr{O}\left(\mathscr{K}_{i}\right)
\end{array}\right\}
$$

The set $\mathscr{O}(\mathscr{K})$ is finite because we consider only finite, acyclic terms.

An occurrence in $\mathscr{K}$ determines a subterm and a functor or variable in $\mathscr{K}$.

Definition 5.2. For a (possibly nonground) term $\mathscr{K}$ and $s \in \mathscr{O}(\mathscr{K})$, the subterm $\mathscr{K} / s$ and functor or variable $\mathscr{K}[s]$ determined by $s$ are defined as follows.

$$
\begin{gathered}
\mathscr{K} / s=\text { if }(s=\epsilon) \text { then } \mathscr{K} \text { else } \mathscr{K}_{i} / s^{\prime}, \text { where }\left(s=i . s^{\prime}\right) \text { and }\left(\mathscr{K} \equiv f\left(\mathscr{K}_{1}, \ldots, \mathscr{K}_{c}\right)\right) \\
\mathscr{K}[s]=\text { if }(\mathscr{K} / s \in \text { Vars }) \text { then } \mathscr{K} / s \text { else } f, \text { where }\left(\mathscr{K} / s \equiv f\left(\mathscr{K}_{1}, \ldots, \mathscr{K}_{c}\right)\right)
\end{gathered}
$$

We next define the TermShift operation which is a closure operation on either sets of occurrences or sets of pairs of occurrences. We use it in correspondence with the idea that if two terms occupy the same storage cells in an implementation, then their corresponding subterms also occupy the same storage cells, and similarly if a term is live at some program point, then its subterms are live too. 
Definition 5.3. For $\mathscr{H}$ a term, $\mathrm{L}_{\mathscr{H}}$ a subset of $\mathscr{O}(\mathscr{K})$, and $\mathrm{P}_{\mathscr{R}}$ a set of ordered pairs $(r, s)$ such that $r, s \in \mathscr{O}(\mathscr{K})$ and $\mathscr{K} / r \equiv \mathscr{K} / s$, we define

$$
\begin{gathered}
\operatorname{TermShift}\left(\mathscr{K}, \mathrm{L}_{\mathscr{H}}\right)=\left\{r . t \mid r \in \mathrm{L}_{\mathscr{H}} \& r . t \in \mathscr{O}(\mathscr{K})\right\}, \\
\operatorname{TermShift}\left(\mathscr{K}, \mathrm{P}_{\mathscr{K}}\right)=\left\{(r . t, s . t) \mid(r, s) \in \mathrm{P}_{\mathscr{H}} \& r . t, s . t \in \mathscr{O}(\mathscr{K})\right\} .
\end{gathered}
$$

(Note, $\mathscr{K} / r \equiv \mathscr{K} / s \Rightarrow(r . t \in \mathscr{O}(\mathscr{K}) \Leftrightarrow s . t \in \mathscr{O}(\mathscr{K}))$.)

Some subterms cannot share their representation, while in the implementations for which our analysis is intended, multiple occurrences of the same free variable always share their representation. So, we introduce two concepts to characterize meaningful sets of sharing pairs for a given term $\mathscr{K}$.

Definition 5.4. For a term $\mathscr{K}$, we call a set $\mathrm{P}_{\mathscr{H}}$ of ordered pairs $(r, s)$ such that $r, s \in \mathscr{O}(\mathscr{K})$, a presharing component for $\mathscr{\mathscr { K }}$ if the following conditions are met.

(1) $\mathrm{P}_{\mathscr{H}}$ is an equivalence relation over $\mathscr{O}(\mathscr{K})$ (i.e., reflexive, symmetric, transitive).

(2) $(r, s) \in \mathrm{P}_{\mathscr{K}} \Rightarrow \mathscr{K} / r \equiv \mathscr{K} / s$ (i.e., only identical subterms can share).

(3) $(r, s) \in \mathrm{P}_{\mathscr{H}} \Rightarrow \operatorname{TermShift}(\mathscr{K},\{(r, s)\}) \subseteq \mathrm{P}_{\mathscr{H}}$ (i.e., the set is closed under TermShift).

Definition 5.5. For a term $\mathscr{K}$, we call a set $\mathrm{P}_{\mathscr{H}}$ of ordered pairs $(r, s)$ such that $r, s \in \mathscr{O}(\mathscr{K})$ a concrete sharing component for $\mathscr{K}$ if $\mathrm{P}_{\mathscr{K}}$ is a presharing component for $\mathscr{K}$, and it satisfies the following condition, which corresponds to the property that multiple occurrences of a free variable share their representation.

$$
(\mathscr{K} / r \equiv \mathscr{K} / s) \&(\mathscr{K} / r \in \text { Vars }) \Rightarrow(r, s) \in \mathrm{P}_{\mathscr{H}} .
$$

We will need two auxiliary operations to compute the symmetric and transitive closure of sets of ordered pairs.

Definition 5.6. For a set $\mathrm{P}_{\mathscr{H}}$ of ordered pairs $(r, s)$, we define

$$
\begin{aligned}
& \text { SymmetricClosure }\left(\mathrm{P}_{\mathscr{Z}}\right)=\left\{(r, s) \mid(r, s) \in \mathrm{P}_{\mathscr{R}} \vee(s, r) \in \mathrm{P}_{\mathscr{X}}\right\}, \\
& \text { TransitiveClosure }\left(\mathrm{P}_{\mathscr{H}}\right)=\left\{\begin{array}{l|l}
\left(r_{1}, s_{n}\right) & \begin{array}{l}
\exists\left(r_{1}, s_{1}\right) \ldots\left(r_{n}, s_{n}\right) \text { a finite } \\
\text { sequence over } \mathrm{P}_{\mathscr{Z}} \text { such that } \\
\left(\forall \ell \in \mathbb{N}: 1 \leq \ell<n \Rightarrow s_{\ell}=r_{\ell+1}\right)
\end{array}
\end{array}\right\} .
\end{aligned}
$$

We also introduce a LiveClosure operation to make explicit the live occurrences implied by a sharing component.

Definition 5.7. For a concrete term $\mathscr{K}$, a set $\mathrm{L}_{\mathscr{K}} \subseteq \mathscr{G}(\mathscr{H})$ and $\mathrm{P}_{\mathscr{\mathscr { R } ^ { \prime }}}$ a set of ordered pairs $(r, s)$ such that $r, s \in \mathscr{O}(\mathscr{K})$, we define

$$
\text { LiveClosure }\left(\mathrm{L}_{\mathscr{H}}, \mathrm{P}_{\mathscr{R}}\right)=\left\{s \mid s \in \mathrm{L}_{\mathscr{H}} \vee\left(\exists r \in \mathrm{L}_{\mathscr{H}}:(r, s) \in \mathrm{P}_{\mathscr{R}}\right)\right\} \text {. }
$$

We now discuss the abstract counterparts of the previous concepts and operations. A set of concrete term environments can be approximated by a ACM Transactions on Programming Languages and Systems, Vol 16, No 2, March 1994. 
type graph. The notions of occurrence and subterm for concrete terms are extended to type graphs, yielding selectors and subgraphs. A selector is a sequence of ordered pairs $\langle i, l\rangle$. Each pair tells how to move from one non-Or node to another: $i$ is an integer selecting a child of the first node, and $l$ is a non-Or label selecting a (unique) principal node of that child.

We assume the following notations, which are formalized in Mulkers [1991].

$-\mathscr{S} \mathscr{T})$ : the set of all selectors in type graph $\mathscr{T}($ similar to $\mathscr{O}(\mathscr{K}))$. $\mathscr{S}(\mathscr{T})$ can be infinite when $\mathscr{T}$ contains back-arcs.

$-\mathscr{T} / S$ : selects a subgraph of $\mathscr{T}$ (similar to $\mathscr{K} / s$ in term $\mathscr{K}$ ). ${ }^{4}$

$\longrightarrow \mathscr{T}[S]$ : selects a node of $\mathscr{T}$ (similar to $\mathscr{H}[s]$ in term $\mathscr{K}$ ).

-TGShift( $\left.\mathscr{T}, \mathrm{E}_{\mathscr{T}}\right)$ : the type graph analogue of TermShift $\left(\mathscr{K}, \mathrm{E}_{\mathscr{H}}\right.$ ). Takes a set (of pairs) of nodes and returns a set (of pairs) of nodes.

- Sel $(\mathscr{K}, s)$ : translates the term occurrence $s$ for a term $\mathscr{K}$ into a type graph selector, e.g., $\operatorname{Sel}(\langle b, f(a, g(c))\rangle, 2.2)=\langle 2, f\rangle .\langle 2, g\rangle$.

Further for the abstract data type of abstract term environments, we assume operations TGSimpleUnify, TGFunctorUnify, TGRestrict, and TGExtend. It is beyond the present scope to discuss in detail these type graph operations on which the specifications of the operations for liveness analysis are based. (See Janssens [1990] and Janssens and Bruynooghe [1990].) In the sequel, we will also use the concretization function TGEnvConc that yields the set of concrete terms described by an abstract term environment $\mathscr{T}^{e}$.

We use sets of nodes and sets of pairs of nodes to associate liveness and sharing information with the abstract term environments. We do not introduce an abstract counterpart for presharing components, but only for concrete sharing components.

Definition 5.8. For an abstract term environment $\mathscr{T}^{e}$ containing the type graph $\mathscr{T}=\left\langle\right.$ Nodes $_{\mathscr{T}}$, ForwardArcs ${ }_{\mathscr{G}}$, BackArcs $\left._{\mathscr{T}}\right\rangle$, we call a symmetric set of ordered pairs $(m, n)$ such that $m, n \in \operatorname{Nodes}_{\mathscr{F}}$, Label $(m)=\operatorname{Label}(n)$, and Label $(m) \neq O r$ an abstract sharing component for $\mathscr{T}$.

An abstract sharing component, ASharing $\mathscr{\mathscr { T }}$, for a type graph $\mathscr{T}$, constrains the sharing that may be possible in the terms represented by the type graph $\mathscr{T}$. Unlike concrete sharing components, which are TermShift closed, abstract sharing components need not be closed with respect to TGShift. The concretization function (Definition 5.11) assures a correct interpretation by first calling the TGShift operation. Further, while a concrete sharing component contains pairs of occurrences indicating identical subterms, the definition of an abstract sharing component requires only that sharing arcs connect nodes having the same label. We will come back to this in Section 7. For the formal

\footnotetext{
${ }^{4}$ An algorithm for constructing the selected subgraph is in Janssens and Bruynooghe [1992]. In some cases, nodes are duplicated.
} 
definition of the alternating-closure operation, AlternatingClosure $(B, C)$, used to mimic the propagation of sharing in the abstract case we refer the reader to Mulkers [1991] and Mulkers et al. [1993].

Having introduced the basic concepts, we are now in the position to formally define the concrete and abstract domains that we consider promising for the purpose of liveness analysis. As was roughly sketched in the previous sections, we instrument the standard concrete domain such that it captures the sharing and liveness of structures. More in particular, we add a twofold sharing component to the term environments in order to maintain detailed information about what sharings are passed down from a calling environment and what sharings are created in a current environment. We further add a liveness component to the term environments that, if propagated through the sharing components, represents the globally live subterms of the environment. A concrete liveness component is closed with respect to the first sharing component and also with respect to the TermShift operation.

Definition 5.9. A concrete liveness environment has the form $\left\langle\mathscr{K},\left\langle\mathrm{CShr}_{\mathscr{Z}}^{0}, \mathrm{CShr}_{\mathscr{H}}^{n}\right\rangle, \mathrm{Clive}_{\mathscr{H}}\right\rangle . \mathscr{K}$ is a term that has principal functor \langle\rangle . The sharing component $\left\langle\mathrm{CShr}_{\mathscr{K}}^{0}, \mathrm{CShr}_{\mathscr{\mathscr { N }}}^{n}\right\rangle$ is such that $\mathrm{CShr}_{\mathscr{\mathscr { H }}}^{0}, \mathrm{CShr}_{\mathscr{M}^{\prime}}^{n}$ are presharing components for $\mathscr{K}$, and TransitiveClosure $\left(\mathrm{CShr}_{\mathscr{R}}^{0} \cup \mathrm{CShr}_{\mathscr{K}}^{n}\right)$ is a concrete sharing component for $\mathscr{K}$. The liveness component CLive $\mathscr{H}_{\mathscr{R}}$ is a subset of $\mathscr{O}(\mathscr{H})$ closed under TermShift, i.e., CLive $\mathscr{H}_{\mathscr{K}}=\operatorname{TermShift}\left(\mathscr{K}\right.$, CLive $\left._{\mathscr{H}}\right)$, and closed with respect to $\mathrm{CShr}_{\mathscr{H}}^{0}$, i.e., CLive $\mathscr{\mathscr { H }}_{\mathscr{T}}=\operatorname{LiveClosure}\left(\mathrm{CLive}_{\mathscr{K}}, \mathrm{CShr}_{\mathscr{H}}^{0}\right)$. We call CLive $\mathscr{\mathscr { R }}$ a concrete liveness component for $\left\langle\mathscr{H},\left\langle\operatorname{CShr}_{\mathscr{H}}^{0}, \operatorname{CShr}_{\mathscr{R}}^{n}\right\rangle\right\rangle$.

Definition 5.10. An abstract liveness environment has the form $\left\langle\mathscr{T}^{e}\right.$, $\left.\left\langle\operatorname{AShr}_{\mathscr{F}}^{0}, \mathrm{AShr}_{\mathscr{T}}^{n}\right\rangle, \mathrm{ALive}_{\mathscr{T}}\right\rangle$ where $\mathscr{T}^{e}$ is an abstract term environment containing type graph $\mathscr{T}=\left\langle\right.$ Nodes $_{\mathscr{F}}$, ForwardArcs $_{\mathscr{F}}$, BackArcs $\left.\mathscr{F}_{\mathscr{F}}\right\rangle$ with \langle\rangle or $\perp$ labeling Root( $\mathscr{T})$. The sharing component $\left\langle\mathrm{AShr}_{\mathscr{T}}^{0}, \mathrm{AShr}_{\mathscr{T}}^{n}\right\rangle$ is such that AlternatingClosure(TGShift $\left(\mathscr{T}, \mathrm{AShr}_{\mathscr{F}}^{0}\right)$, $\left.\operatorname{TGShift}_{(\mathscr{T}}, \mathrm{AShr}_{\mathscr{F}}^{n}\right)$ ), and $\mathrm{AShr}_{\mathscr{T}}^{0}, \mathrm{AShr}_{\mathscr{I}}^{n}$ are abstract sharing components for $\mathscr{T}$. The liveness component ALive is $_{\text {a subset of }}$ Nodes $_{\mathscr{T}}$, consisting of nodes not labeled Or. We call ALive $_{\mathscr{T}}$ an abstract liveness component for $\left\langle\mathscr{T}^{e},\left\langle\mathrm{AShr}_{\mathscr{J}}^{0}, \mathrm{AShr}_{\mathscr{g}}^{n}\right\rangle\right\rangle$.

The abstract interpretation procedure computing abstract AND-OR graphs will associate with every program point an abstract liveness environment. The component $\mathrm{CShr}_{\mathscr{H}}^{0}$ (resp., AShr $r_{\mathscr{P}}^{0}$ ) stands for the (old) sharing pushed down from the calling environment to the current environment (associated with the clause being interpreted); the component CShr $r_{\mathscr{T}}^{n}$ (resp., AShr $r_{\mathscr{T}}^{n}$ ) stands for the (new) sharing created in the current environment up to the current program point. So, the complete set of sharing arcs for a program point is obtained by the TransitiveClosure (resp., AlternatingClosure) operation. A correct interpretation of the liveness component requires that the sharing relation is taken into account to derive from the component CLive $_{\mathscr{K}}$ (resp., ALive $_{\mathscr{T}}$ ) the full set of globally live terms, i.e., live with respect to some calling environment. The information that a compiler needs at a program point to decide upon the liveness of some term also requires deriving the set of locally live terms. 
Note: In the sequel when there is no confusion possible, we use CSharing ${ }_{\mathscr{K}}$ to denote either TransitiveClosure $\left(\mathrm{CShr}_{\mathscr{H}}^{0} \cup \mathrm{CShr}_{\mathscr{H}}^{n}\right)$ or $\left\langle\mathrm{CShr}_{\mathscr{F}}^{0}, \mathrm{CShr}_{\mathscr{\mathscr { K }}}^{n}\right\rangle$, and

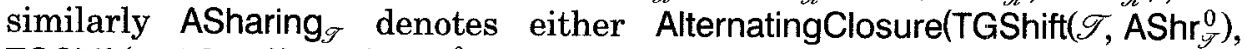
TGShift( $\left.\left.\mathscr{T}, A S h r_{\mathscr{T}}^{n}\right)\right)$, or $\left\langle\mathrm{AShr}_{\mathscr{G}}^{0}, \mathrm{AShr} r_{\mathscr{J}}^{n}\right\rangle$. It will be clear from the context what is meant.

An abstract liveness environment, consisting of a type graph and sharing and liveness components, characterizes the current binding environment in a Prolog-style computation. The concretization function LiveEnvConc for abstract liveness environments is defined as follows.

Definition 5.11. For an abstract liveness environment $\left\langle\mathscr{T}^{e},\left\langle\mathrm{AShr}_{\mathscr{F}}^{0}, \mathrm{AShr}_{\mathscr{T}}^{n}\right\rangle\right.$, ALive $\left._{\mathscr{T}}\right\rangle$,

LiveEnvConc $\left(\left\langle\mathscr{T}^{e},\left\langle\mathrm{AShr}_{\mathscr{T}}^{0}, \mathrm{AShr}_{\mathscr{T}}^{n}\right\rangle\right.\right.$, ALive $\left.\left._{\mathscr{T}}\right\rangle\right)=$

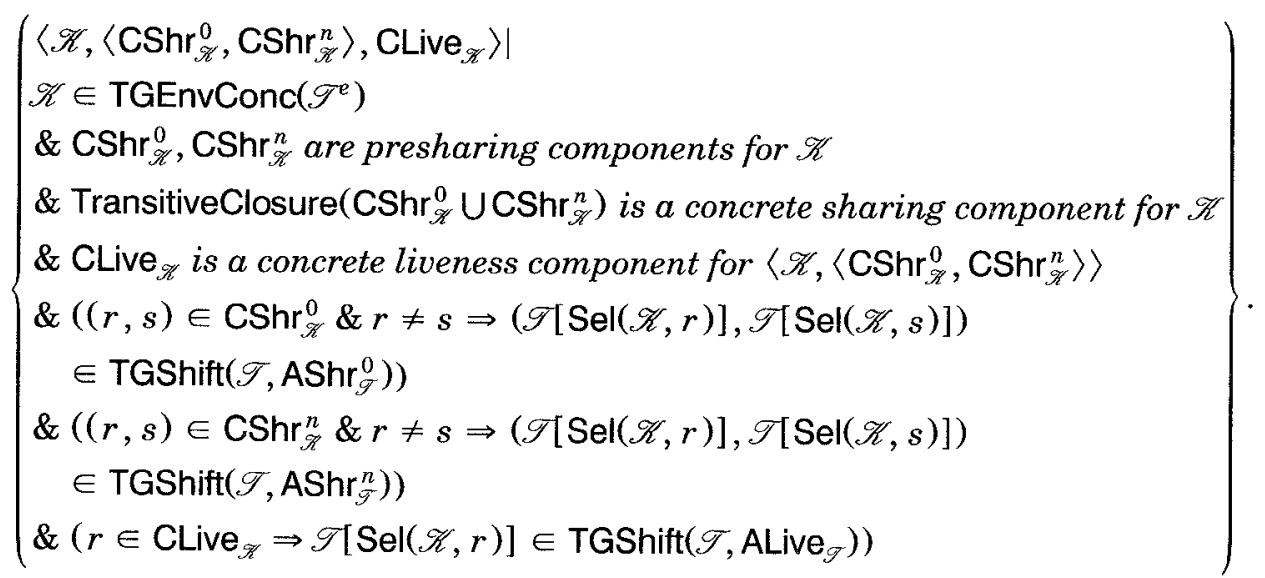

Note that the concrete reflexive pairs do not take part in the mapping because they are in fact meaningless.

5.4.2 Primitive Operations. We will formalize only the operation for simple unification. For the other operations, AbstrFunctorUnify, AbstrRestrict, and AbstrExtend, we refer the interested reader to Mulkers [1991] and Mulkers et al. [1993].

Consider the operation SimpleUnify $\left\langle\mathscr{K}_{l n}\right.$, CSharing $_{\mathscr{H}_{2 n}}$, CLive $\left.\left._{\mathscr{K}_{2 n}}\right\rangle, i, j\right)$ for unifying the $i$ th and $j$ th terms in $\mathscr{K}_{i n}$ in the presence of the sharing component, CSharing $\mathscr{\mathscr { H }}_{2 n}$, and liveness component, CLive $\mathscr{\mathscr { H }}_{i n}$. If we assume that $\mathscr{K}_{i n} / \ell$ is the binding of program variable $X_{\ell}$, for each $\ell, 1 \leq \ell \leq \operatorname{arity}\left(\mathscr{K}_{i n}[\epsilon]\right)$, then SimpleUnify $\left(\left\langle\mathscr{K}_{l n}\right.\right.$, CSharing $_{\mathscr{K}_{l n}}$, CLive $\left.\left._{\mathscr{K}_{l n}}\right\rangle, i, j\right)$ corresponds to the basic operation $X_{\iota}=X_{j}$.

Consider SimpleUnify $\left\langle\mathscr{K}_{i n}\right.$, CSharing $\mathscr{\mathscr { R }}_{i n}$, CLive $\left.\left._{\mathscr{K}_{l n}}\right\rangle, i, j\right)=\left\langle\mathscr{K}_{\text {out }}\right.$, CSharing $_{\mathscr{H}_{\text {out }}}$, CLive $\left._{\mathscr{K}_{\text {out }}}\right\rangle^{\prime}$. The term part $\left(\mathscr{K}_{\text {out }}\right.$ ) as well as the sharing (CSharing ${ }_{K_{\text {out }}}$ ) and liveness part $\left(\right.$ CLive $\left._{\mathscr{X}_{0 u t}}\right)$ must reflect the effect of unification. For the term part, this means applying the most general unifier of $\mathscr{K}_{l n} / i$ and $\mathscr{K}_{n n} / j$, 
defined, for example, as in Lassez et al. [1988] and denoted by mgu $\left(\mathscr{K}_{n n} / i, \mathscr{K}_{n n} / j\right)$. For the sharing part expressing the effect of unification means adding pairs indicating positions where unification has introduced new sharing of subterms. Recall that the language implementations that we address create new sharing when binding a variable to an existing structure. The sharing existing in the input term prior to the unification is preserved and extended downward in the case that the term is further instantiated by the unification (cfr., the TermShift operation). Finally, the new sharing propagates through the old sharing.

Also the liveness part has to be extended downward in the case that the term is further instantiated by the unification (i.e., the unification operation has to ensure that the liveness component of the concrete environment remains closed with respect to the TermShift operation (see Definition 5.9)). Changes in the global liveness of terms that are due to an update of the sharing component need not be made explicit.

Definition 5.12. For a concrete liveness environment $\left\langle\mathscr{K}_{i n},\left\langle\operatorname{CShr}_{\mathscr{H}_{i n}}^{0}\right.\right.$, $\left.\mathrm{CShr}_{\mathscr{R}_{i n}}^{n}\right\rangle$, CLive $\left.\mathscr{\mathscr { R }}_{l n}\right\rangle$, and $i$ and $j$ such that $1 \leq i<j \leq \operatorname{arity}\left(\mathscr{K}_{i n}[\epsilon]\right)$,

$$
\begin{aligned}
& \text { SimpleUnify }\left(\left\langle\mathscr{K}_{\imath n},\left\langle\operatorname{CShr}_{\mathscr{M}_{\imath n}}^{0}, \operatorname{CShr}_{\mathscr{R}_{l n}}^{n}\right\rangle, \text { CLive }_{\mathscr{K}_{\imath n}}\right\rangle, i, j\right)= \\
& \begin{cases}\text { if } & \operatorname{mgu}\left(\mathscr{K}_{\text {in }} / i, \mathscr{K}_{\text {in }} / j\right) \text { is fail } \\
\text { then } & \text { fail } \\
\text { else } & \left\langle\mathscr{K}_{\text {out }},\left\langle\mathrm{CShr}_{\mathscr{K}_{\text {out }}}^{0}, \mathrm{CShr}_{\mathscr{K}_{\text {out }}}^{n}\right\rangle, \mathrm{CLive}_{\mathscr{\mathscr { T }}_{\text {out }}}\right\rangle,\end{cases}
\end{aligned}
$$

where

$$
\mathscr{K}_{\text {out }} \equiv \mathscr{K}_{i n} \sigma, \quad \text { for } \sigma=\operatorname{mgu}\left(\mathscr{K}_{\imath n} / i, \mathscr{K}_{\text {} n} / j\right)
$$

and

$$
\begin{aligned}
& \operatorname{CShr}_{\mathscr{T}_{\text {out }}}^{0}=\operatorname{TermShift}\left(\mathscr{K}_{\text {out }}, \operatorname{CShr}_{\mathscr{R}_{i n}}^{0}\right) \text {, } \\
& \text { AccNew }=\operatorname{TermShift}\left(\mathscr{K}_{\text {out }}, \operatorname{CShr}_{\mathscr{R}_{2 n}}^{n}\right) \text {, } \\
& \text { New } \left.=\text { TermShift }\left(\mathscr{K}_{\text {out }}, \text { SymmetricClosure(S-BindingPairs }\left(\mathscr{K}_{i n}, i, j\right)\right)\right), \\
& \mathrm{CShr}_{\mathscr{M}_{0 u t}}^{n}=\text { TransitiveClosure(AccNewUNew), } \\
& \text { CLive }_{\mathscr{A}_{\text {out }}}=\operatorname{TermShift}\left(\mathscr{H}_{\text {out }}, \text { CLive }_{\mathscr{L}_{l n}}\right) \text {, }
\end{aligned}
$$

with

$$
\text { S-BindingPairs }\left(\mathscr{K}_{i n}, i, j\right)=\left\{(i . p, j . p) \mid \begin{array}{l}
i \cdot p, j \cdot p \in \mathscr{\mathscr { C }}\left(\mathscr{K}_{\imath n}\right) \& \\
\left(\mathscr{K}_{\imath n} / i . p \in \operatorname{Vars} \vee \mathscr{K}_{i n} / j . p \in \operatorname{Vars}\right)
\end{array}\right\} .
$$


For example, consider the following concrete liveness environment which corresponds to Figure 9(a) (Section 5.1). Note that for reasons of compactness, we leave out the reflexive and symmetric pairs from the sharing components.

$$
\begin{aligned}
\mathscr{K}_{i n} & =\langle\mathrm{h}(\mathrm{X}, \mathrm{f}(\mathrm{Z})), \mathrm{h}(\mathrm{g}(\mathrm{Y}), \mathrm{Y})\rangle \\
\mathrm{CShr}_{\mathscr{K}_{i n}}^{0} & =\{(2.1 .1,2.2)\} \\
\mathrm{CShr}_{\mathscr{H}_{i n}}^{n} & =\varnothing \\
\mathrm{CLive}_{\mathscr{K}_{i n}} & =\{1.2 .1\}
\end{aligned}
$$

When we compute SimpleUnify $\left(\left\langle\mathscr{K}_{i n}\right.\right.$, CSharing $\left.\left._{\mathscr{K}_{i n}}, \mathrm{CLive}_{\mathscr{H}_{i n}}\right\rangle, 1,2\right)$, a set SBindingPairs is constructed by locating each variable occurrence in the input terms that are to be unified, $\mathscr{K}_{i n} / \mathbf{1}$ and $\mathscr{K}_{i n} / 2$, and by introducing a pair giving the position of that variable occurrence and the corresponding position in the other term being unified (the other of $\mathscr{K}_{i n} / 1$ and $\mathscr{K}_{i n} / 2$ ).

$$
\text { S-BindingPairs }\left(\mathscr{K}_{i n}, 1,2\right)=\{(1.1,2.1),(1.2,2.2)\}
$$

The sets $\operatorname{CShr}_{\mathscr{K}_{i n} n}^{0}, \operatorname{CShr}_{\mathscr{K}_{i n}}^{n}$, and S-BindingPairs $\left(\mathscr{K}_{2 n}, 1,2\right)$ are shifted according to the instantiated term $\mathscr{K}_{\text {out }}$. The result is given by the components $\mathrm{CShr}_{\mathscr{K}_{\text {out }}}^{0}$, AccNew, and New respectively. The function TransitiveClosure is used to combine the sharing introduced by the present unification with the sharing that was newly introduced at the present level in the AND-OR graph.

$$
\begin{aligned}
& \mathscr{K}_{\text {out }}=\langle\mathrm{h}(\mathrm{g}(\mathrm{f}(\mathrm{Z})), \mathrm{f}(\mathrm{Z})), \mathrm{h}(\mathrm{g}(\mathrm{f}(\mathrm{Z})), \mathrm{f}(\mathrm{Z}))\rangle \\
& \mathrm{CShr}_{\mathscr{S}_{\text {out }}}^{0}=\operatorname{CShr}_{\mathscr{\mathscr { N }}_{\text {in }}}^{0} \cup\{(2.1 .1 .1,2.2 .1)\} \\
& \text { AccNew }=\varnothing \\
& \text { New }=\text { S-BindingPairs }\left(\mathscr{K}_{i n}, 1,2\right) \cup \\
& \{(1.1 .1,2.1 .1),(1.1 .1 .1,2.1 .1 .1),(1.2 .1,2.2 .1)\} \\
& \mathrm{CShr}_{\mathscr{\mathscr { x }}_{\text {out }}}^{n}=\mathrm{New} \\
& \text { CLive }_{\mathscr{K}_{\text {out }}}=\text { CLive }_{\mathscr{H}_{\text {In }}}
\end{aligned}
$$

Finally the function TransitiveClosure is also needed to generate the full sharing component by propagating the new sharing according to the old sharing existing in the terms.

$$
\begin{aligned}
& \text { CSharing }_{\mathscr{R}_{\text {out }}}=\operatorname{TransitiveClosure}\left(\mathrm{CShr}_{\mathscr{R}_{\text {out }}}^{0} \cup \operatorname{CShr}_{\mathscr{\mathscr { H }}_{\text {out }}}^{n}\right) \\
& =\operatorname{CShr}_{\mathscr{H}_{\text {out }}}^{0} \cup \operatorname{CShr}_{\mathscr{H}_{\text {out }}}^{n} \cup\{(1.1 .1,1.2),(1.1 .1,2.2),(1.2,2.1 .1) \text {, } \\
& (1.1 .1 .1,1.2 .1),(1.1 .1 .1,2.2 .1),(1.2 .1,2.1 .1 .1)\} \text {. }
\end{aligned}
$$

The full set of live nodes is obtained as (see Figure 9(b), Section 5.1)

$$
\text { LiveClosure }\left(\text { CLive }_{\mathscr{K}_{\text {out }}} \text {, CSharing } \mathscr{\mathscr { H }}_{\text {out }}\right)=\{1.2 .1,2.2 .1,1.1 .1 .1,2.1 .1 .1\} \text {. }
$$

The main difference between the concrete and abstract unification operations is that the abstract unification uses AlternatingClosure, rather than TransitiveClosure, to propagate the sharing relation. The definition of the abstract operation further uses auxiliary functions Convert and S-BindingEdges, defined below. 
Definition 5.13. For an abstract liveness environment $\left\langle\mathscr{F}_{i n}^{e},\left\langle\mathrm{AShr}_{\mathscr{J}_{i n}}^{0}\right.\right.$, $\left.\operatorname{AShr}_{\mathscr{F}_{i n}}^{n}\right\rangle$, ALive $\left._{\mathscr{T}_{1 n}}\right\rangle$, and $i$ and $j$ such that $1 \leq i<j \leq \operatorname{arity}\left(\mathscr{T}_{i n}[\epsilon]\right)$,

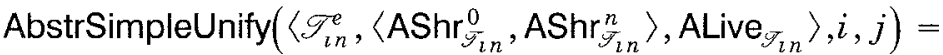

$$
\begin{aligned}
& \begin{cases}\text { if } & \text { TGSimpleUnify }\left(\mathscr{T}_{\text {in }}^{e}, i, j\right) \text { is fail } \\
\text { then } & \text { fail } \\
\text { else } & \left\langle\mathscr{G}_{\text {out }}^{e},\left\langle\mathrm{AShr}_{\mathscr{T}_{\text {out }}}^{0}, \mathrm{AShr}_{\mathscr{J}_{\text {out }}}^{n}\right\rangle, \text { ALive }_{\mathscr{T}_{\text {out }}}\right\rangle,\end{cases}
\end{aligned}
$$

where

$$
\mathscr{Y}_{\text {out }}^{e}=\text { TGSimpleUnify }\left(\mathscr{F}_{2 n}^{e}, i, j\right)
$$

and

$$
\begin{aligned}
& \operatorname{AShr}_{\mathscr{F}_{\text {out }}}^{0}=\operatorname{Convert}\left(\mathscr{T}_{\text {in }}, \mathrm{AShr}_{\mathscr{F}_{i n}}^{0}, \mathscr{T}_{\text {out }}\right), \\
& \mathrm{C}=\operatorname{TGShift}\left(\mathscr{T}_{\text {out }}, \operatorname{Convert}\left(\mathscr{T}_{\text {in }}, \operatorname{AShr}_{\mathscr{G}_{\text {ln }}}^{n}, \mathscr{T}_{\text {out }}\right)\right) \text {, } \\
& \mathrm{B}=\text { TGShift }\left(\mathscr{T}_{\text {out }},\right. \text { SymmetricClosure } \\
& \text { (S-BindingEdges } \left.\left(\mathscr{T}_{i n}, \mathscr{T}_{\text {out }}, i, j\right)\right) \text { ), } \\
& \operatorname{TGShift}\left(\mathscr{T}_{\text {out }}, \mathrm{AShr}_{\widetilde{S}_{\text {out }}}^{n}\right)=\text { AlternatingClosure }(\mathrm{C}, \mathrm{B}) \text {, } \\
& \text { ALive }_{\mathscr{J}_{\text {out }}}=\operatorname{Convert}\left(\mathscr{T}_{l n}, \text { ALive }_{\mathscr{T}_{l n}}, \mathscr{T}_{\text {out }}\right) \text {. }
\end{aligned}
$$

Note that we define AShr ${ }_{\widetilde{S_{0}}}^{n}$ indirectly by specifying its image under TGShift. At intermediate stages, an implementation of the liveness analysis might use more economical sharing components that are not closed with respect to the shift operation.

The function Convert takes an abstract sharing or liveness component for a type graph $\mathscr{T}_{1 n}$ and reexpresses it for a type graph $\mathscr{T}_{\text {out }}$. The terms represented by $\mathscr{T}_{\text {out }}$ can be instantiations of terms represented by $\mathscr{T}_{i n}$, e.g., due to unification. As a consequence, nodes labeled $\mathrm{V}$ in $\mathscr{T}_{i n}$ can correspond to nodes in $\mathscr{T}_{\text {out }}$ not labeled $\mathrm{V}$, indicating a further instantiation of the terms.

Definition 5.14. For two abstract term environments $\mathscr{T}_{i n}^{e}$ and $\mathscr{F}_{\text {out }}^{e}$ such that $\operatorname{arity}\left(\mathscr{T}_{i n}[\epsilon]\right)=\operatorname{arity}\left(\mathscr{T}_{\text {out }}[\epsilon]\right)$, and an abstract sharing component $\mathrm{P}_{\mathscr{T}_{i n}}$ for $\mathscr{T}_{i n}$, define

Convert $\left(\mathscr{T}_{\text {in }}, \mathrm{P}_{\mathscr{S}_{\text {In }}}, \mathscr{T}_{\text {out }}\right)=$

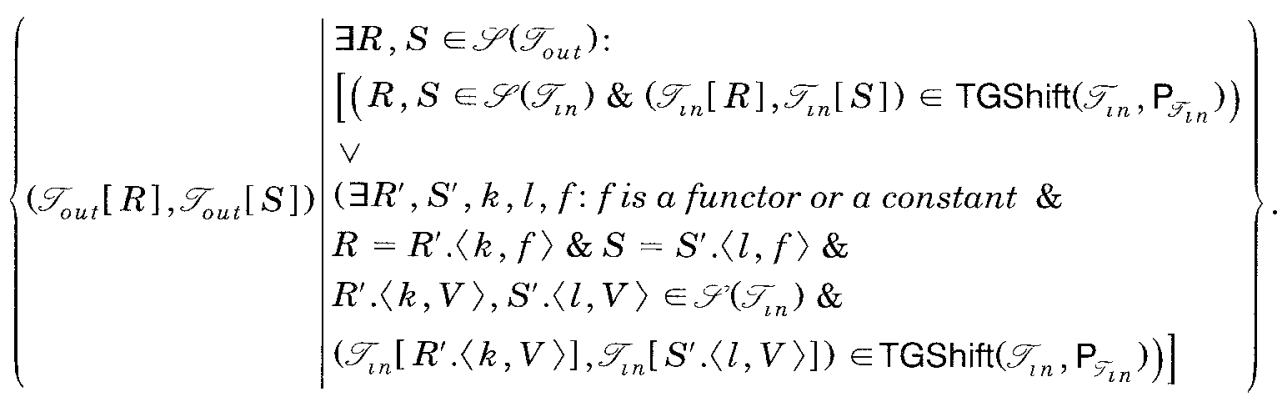

ACM Transactions on Programming Languages and Systems, Vol. 16, No 2, March 1994. 
The generalization of the function Convert for liveness components is straightforward. The function S-BindingEdges is the type graph analogue of the S-BindingPairs function for concrete terms.

Definition 5.15. For an abstract term environment $\mathscr{T}_{i n}^{e}$, and $i$ and $j$ such that $1 \leq i<j \leq \operatorname{arity}\left(\mathscr{T}_{i n}[\epsilon]\right)$ and $\mathscr{T}_{\text {out }}^{e}=\operatorname{TGSimpleUnify}\left(\mathscr{T}_{i n}^{e}, i, j\right)$,

$$
\begin{aligned}
& \text { S-BindingEdges }\left(\mathscr{T}_{\text {tn }}, \mathscr{T}_{\text {out }}, i, j\right)= \\
& \left\{\begin{array}{ll}
\left(T_{\text {out }}[R], \mathscr{T}_{\text {out }}[S]\right) & \begin{array}{l}
\exists R, S \in \mathscr{S}\left(\mathscr{T}_{\text {out }}\right), \exists f: \text { fis a functor, a constant or } V \& \\
{[(R=\langle i, f\rangle \& S=\langle j, f\rangle \&} \\
\left.\left(\langle i, V\rangle \in \mathscr{S}\left(\mathscr{T}_{\text {in }}\right) \vee\langle j, V\rangle \in \mathscr{S}\left(\mathscr{T}_{\text {in }}\right)\right)\right) \\
\left(\text { either the } i \text { th or the } j \text { th component of } \mathscr{T}_{\text {in }}\right. \text { is itself } \\
\vee \\
(\exists g, T, l: R=\langle i, g\rangle . T .\langle l, f\rangle \& S=\langle j, g\rangle . T .\langle l, f\rangle \& \\
\left.\left(\langle i, g\rangle . T \cdot\langle l, V\rangle \in \mathscr{S}\left(\mathscr{T}_{\text {in }}\right) \vee\langle j, g\rangle . T .\langle l, V\rangle \in \mathscr{S}\left(\mathscr{T}_{\text {in }}\right)\right)\right) \\
(\text { or there is a V-node inside the ith or } j \text { th component } \\
\text { of } \left.\left.\mathscr{T}_{\text {in }}\right)\right]
\end{array}
\end{array}\right\} .
\end{aligned}
$$

Example. Figure 24(a) represents a type graph $\mathscr{T}$, abstract sharing components $\mathrm{AShr}_{\mathscr{g}}^{0}=\varnothing$ and AShr $\mathrm{T}_{\mathscr{T}}^{n}=\{(m 1, m 8),(m 9, m 10)\}$, and liveness component $\mathrm{ALive}_{\mathscr{T}}=\{m 1\}$. Globally live nodes are marked ${ }^{* *}$; sharing arcs are represented by means of dotted arcs. For the nodes of the type graph, we show both their name (e.g., $m 3$ ) and label (e.g., $f=$ Label $(m 3)$ ). Note that $\langle X, f(X, f(Y, a)), f(a, f(a, a))\rangle$ and $\langle X, f(Y, f(X, a)), f(a, f(a, a))\rangle$ are both represented by $\left\langle\mathscr{F}^{e}\right.$, ASharing $_{\mathscr{T}}$, ALive $\left._{\mathscr{T}}\right\rangle$, but $\langle X, f(X, f(X, a)), f(a, f(a, a))\rangle$ is not, because $m 8$ is not self-sharing. To compute AbstrSimpleUnify $\left\langle\mathscr{T}^{e}\right.$, ASharing $_{\mathscr{T}}$, ALive $\left.\left._{\mathscr{T}}\right\rangle, 2,3\right)$, we use TGSimpleUnify $\left(\mathscr{T}^{e}, 2,3\right)$ to construct the output type graph $\mathscr{T}^{\text {(see }}$ Figure 24(b)). Then we compute

$$
\begin{gathered}
\text { Convert } \left.\mathscr{T}, \operatorname{AShr}_{\mathscr{T}}^{n}, \mathscr{T}\right)=\{(n 5, n 6),(n 5, n 10),(n 12, n 13)\}=\mathrm{C}, \\
\text { S-BindingEdges }(\mathscr{T}, \mathscr{T}, 2,3)=\{(n 6, n 8),(n 10, n 12)\}=\mathrm{B}, \\
\text { AlternatingClosure }(\mathrm{C}, \mathrm{B}) \\
=\mathrm{C} \cup \mathrm{B} \cup\{(n 5, n 8),(n 5, n 12),(n 10, n 13),(n 5, n 13)\}, \\
\text { Convert }\left(\mathscr{T}, \text { ALive }_{\mathscr{T}}, \mathscr{T}\right)=\{n 4, n 5\} .
\end{gathered}
$$

Again, we left out the symmetric arcs (reflexive arcs are not because they are meaningful; however in this example they do not occur). In Figure 24(b) the elements of $B$ are represented as dashed arcs and the elements of $C$ as dotted arcs. Note that the sharing arc $(m 1, m 8)$ in $\mathscr{T}$ corresponds to two sharing arcs $(n 5, n 6),(n 5, n 10)$ in $\mathscr{T}$.

We now formulate the main theorem stating the safety of the abstract unification. 


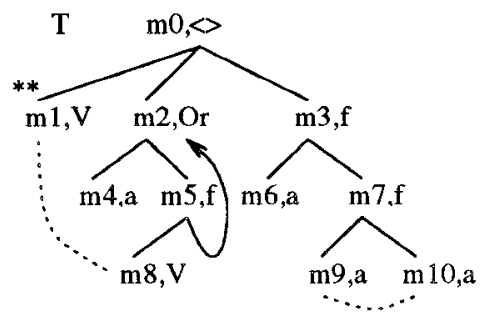

(a)

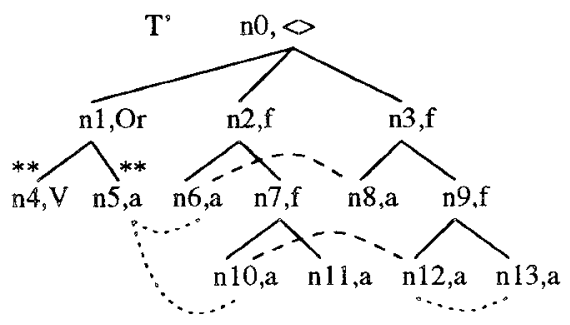

(b)

Fig. 24. Abstract input (a) and output (b) liveness environments for AbstrSımpleUnify.

Theorem SAFETy of AbstrSimpleUnify [Mulkers 1991]. Assuming the following conditions,

(1) $\left\langle\mathscr{T}_{l n}^{e},\left\langle\mathrm{AShr}_{\mathscr{T}_{l n}}^{0}, \mathrm{AShr}_{\widetilde{J}_{l n}}^{n}\right\rangle\right.$, ALive $\left._{\mathscr{F}_{l n}}\right\rangle$ is an abstract liveness environment

(2) $\left\langle\mathscr{K}_{i n},\left\langle\mathrm{CShr}_{\mathscr{H}_{l n}}^{0}, \mathrm{CShr}_{\mathscr{H}_{1 n}}^{n}\right\rangle, \mathrm{CLive}_{\mathscr{H}_{i n}}\right\rangle$ is a concrete liveness environment

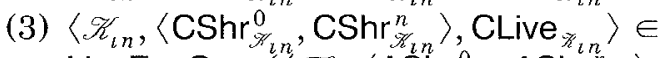

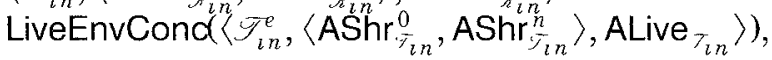

(4) $1 \leq i<j \leq \operatorname{arity}\left(\mathscr{T}_{l n}[\epsilon]\right)=\operatorname{arity}\left(\mathscr{K}_{\imath n}[\epsilon]\right)$

(5) $\left\langle\mathscr{H}_{\text {out }},\left\langle\operatorname{CShr}_{\mathscr{H}_{\text {out }}}^{0}, \operatorname{CShr}_{\mathscr{\mathscr { T }}_{\text {out }}}^{n}\right\rangle, \mathrm{CLive}_{\mathscr{I}_{\text {out }}}\right\rangle=$

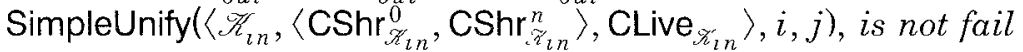

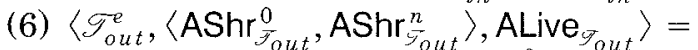
AbstrSimpleUnify $\left.\left\langle\mathscr{T}_{i n}^{e},\left\langle\mathrm{AShr}_{\bar{\zeta}_{l n}}^{0}, \mathrm{AShr}_{\bar{I}_{l n}}^{n}\right\rangle, \mathrm{ALive}_{\bar{\zeta}_{l n}}\right\rangle, i, j\right)$

it follows that

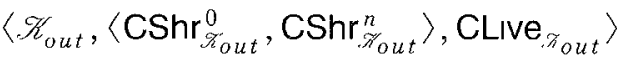

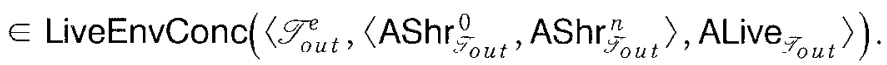

The proof is based on the safety of type graph unification [Janssens and Bruynooghe 1992], the well-definedness of SimpleUnify, and the close relation between the abstract and concrete operations. We showed that each sharing pair created by one of the auxiliary functions TermShift, S-BindingPairs, and TransitiveClosure has an abstract counterpart created by the abstract operations TGShift, S-BindingEdges, and AlternatingClosure and that the abstract counterparts of the initial sharing pairs are preserved by the Convert operation. Similar safety theorems were proved for the other basic operations [Mulkers 1991].

\section{PRACTICAL USEFULNESS OF LIVENESS INFORMATION}

We discuss the usefulness of liveness information to enable reuse of memory cells in structure-copying implementations, either based on the WAM or some other basic instruction set (e.g., Taylor [1991] and Van Roy [1990]). 
Mariën et al. [1989] describe an experiment set up to assess the impact of the information obtained by global-flow analysis on the extent of code optimizations that can be performed by a compiler. Information about liveness of data structures makes it possible to recognize at compile time whether storage occupied by a data structure can be reused to create new data structures in a procedure. When at run time a compound term becomes accessible for the first time, we say the term is being constructed. When a pattern is matched against a compound term that is already accessible, we say the components of the term are being selected. Type and mode analysis in many cases allows us to predict at compile time whether a unification is a selection rather than a construction operation, e.g., if prior to a unification $X=f\left(Y_{1}, \ldots, Y_{n}\right)$, the variable $X$ is still unbound, the unification causes the construction on the heap of a structured term (e.g., the allocation of a record consisting of $n+1$ heap entries). On the other hand, if $X$ is not a free variable but the $Y_{1}, \ldots, Y_{n}$ are, then the unification results in references being created from the variables $Y_{i}$ to the respective subterms of $X$. Selection statements in particular are good candidates to check for the possible creation of garbage cells, i.e., cells that have no further references.

So-called local reuse of garbage cells focuses on programs in which the selection operation that creates garbage is followed by a construction operation within the same clause that requires the same or less amount of storage as released by the selection statement. The experiments discussed consider only a restricted case of local reuse of garbage cells, namely, reuse in a construction operation within the same chunk of the clause where the garbage is created. A chunk is a number, possibly zero, of in-line calls followed by an out-of-line call, and possibly preceded by the head of the clause. In-line calls are calls which use only argument registers of the WAM (e.g., deterministic built-in predicates). Consider the following clause.

$$
\text { append }(X, Y, Z):-X=[E \mid U], Z=[E \mid W], \quad \text { append }(U, Y, W) .
$$

The clause consists of one chunk only. However, for the recursive clause of nrev $/ 2$, the selection and the construction operation belong to different chunks because nrev( $U, R U)$ is an out-of-line call.

$$
\operatorname{nrev}(X, Y):-X=[E \mid U], \operatorname{nrev}(U, R U), \text { Last }=[E], \quad \text { append(RU, Last, } Y) \text {. }
$$

Within a chunk, a temporary register suffices to keep track of the garbage cell until it is needed. In the other cases, a temporary register cannot be used because the contents of such a register may not survive the call of a Prolog procedure. A solution consists in changing the classification of the clause variables, such that a permanent variable on the environment stack keeps the address of the garbage cell until it is needed. The numbers of performance improvement given by Mariën et al. [1989] showed that there is little or no measurable time overhead caused by the new instructions for local reuse and that a significant gain can be expected from avoiding run-time garbage collection.

It is important to note that a correct interpretation of the live-structure information obtained by the analysis is implementation dependent. Indeed, in 

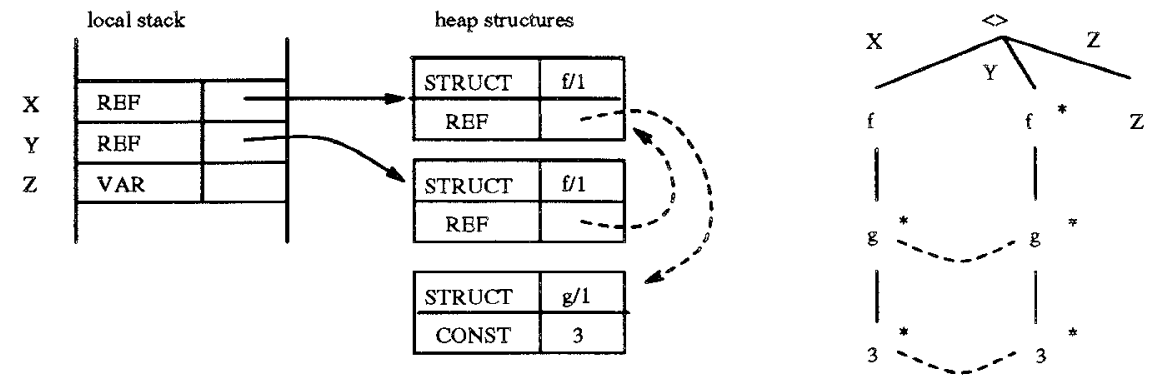

Fig. 25. A reference chain passing through an argument of a dead structure.

WAM-like implementations it is possible that a live argument of a dead structure cell is accessible through a reference chain that passes through an argument field of the structure cell. For instance, consider the following piece of Prolog code:

$$
p:-X=f\left(\_\right), Y=f\left(\_\right), X=Y, X=f(g(3)),(P) Z=h(a), \quad \text { use(Y). }
$$

On the left in Figure 25, we sketch a memory layout that can occur in a real implementation when program point (p) is reached. On the right, the corresponding abstract liveness environment computed by the live-structure analysis is shown. It indicates that the principal functor cell of the structured term that variable $X$ is bound to is dead. Moreover, the next operation $(\mathrm{Z}=\mathrm{h}(\mathrm{a}))$ is known to be a construction needing the same amount of storage. However, because of the reference chain passing through the argument field of the structure cell representing the $f / 1$ functor on the heap, local reuse is not safe. A sufficient condition for safety requires, that in addition to the principal functor being dead, its immediate children should also be dead.

Note that the difficulty is not inherent in all WAM-based implementations, e.g., in the (didactical) PAM-3 machine [Janssens et al. 1987], the problem does not occur because there is an entry for each clause variable in an environment frame on the local stack (all clause variables are permanent variables), and no heap cell ever gets the tag VAR. As a consequence, no reference chain will ever pass through the argument field of a structure cell on the heap.

The safety requirement can be significantly weakened at a program point immediately following a selection operation. It suffices that the functor cell is dead after the selection, and the children corresponding to argument fields that are going to be overwritten are dead, nonvariable, and not shared prior to the selection. To identify the problem cases that can arise, consider Figure 26. At the top, a possible memory layout is shown that holds prior to a selection operation $X=h\left(Y_{1}, Y_{2}, Y_{3}, Y_{4}, Y_{5}, Y_{6}, Y_{7}\right)$. In the middle, the corresponding liveness environment is shown. Note that the $Y_{1}$ variables are marked locally live at the time of the selection. They will cause some of the subterms of $X$ to become live after the selection. Hence, if the stronger condition were used, local reuse of the record would not be considered safe. At 


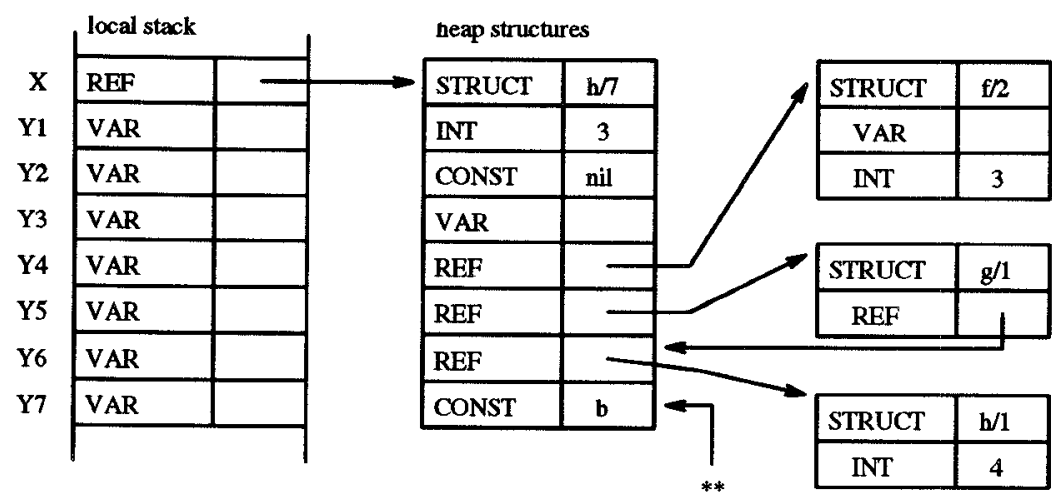
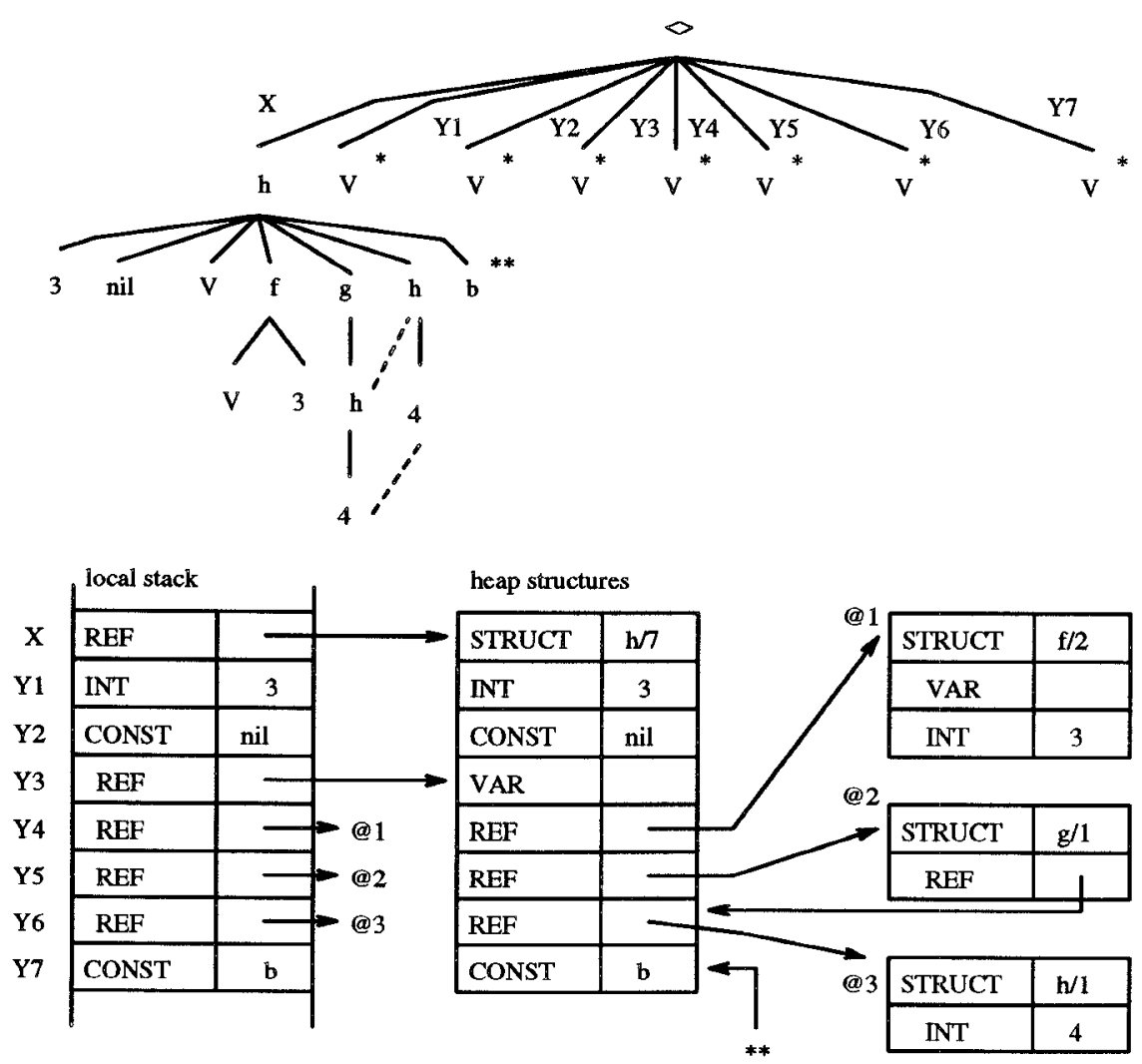

Fig. 26. The effect of a selection in a WAM-based implementation.

the bottom in Figure 26, the situation after the selection is sketched. Note that we assume full dereferencing is performed at a selection operation and that atomic values are copied. The third, sixth, and seventh argument fields of the record representing the $h / 7$ functor are unsafe to overwrite after the selection. The third because it is a variable field referenced by a live program 
variable $Y_{3}$; the sixth because there is a reference chain passing through which starts from a locally live structure bound to $Y_{5}$; and similarly for the seventh argument which may be referenced from a globally live structure. However, the record could still be safely reused in a construction such as $Z=h\left(a, b, Y_{3}, c, d, Y_{6}, Y_{7}\right)$ or $Z=k(a, b)$, for which the unsafe argument fields need not be changed.

In the implementation of Taylor [1991], aliased free variables are represented by a circular list. When a variable becomes instantiated, all the cells in the list of aliases are assigned the same reference pointing to the structure that the variable is unified with. As a consequence, reference chains are never longer than one. So, for the safe reuse of a dead structure cell after a selection operation, it is sufficient to have the children be nonvariable prior to the selection, since references to non-Var terms cannot pass through an argument field of the structure cell.

\section{DESIGN ALTERNATIVES AND COST/PRECISION TRADEOFFS}

An early design decision we made was to separate type analysis (including variable-sharing analysis) from the structure-sharing analysis; i.e., we use the domain of type graphs and its operations as an abstract data type. In this way, the exposition of the live-structure analysis was simplified considerably as was the formal correctness proof of the primitive operations. However, a closer integration of the type and structure-sharing analyses may lead to more precise results and will certainly improve the efficiency of the analysis, as variable sharing is subsumed by structure sharing. The type graph unification algorithm presented by Bruynooghe and Winsborough [1992] is an interesting candidate to form the basis for such an integration.

Another design decision has to do with the properties of the abstract sharing components. While for a concrete sharing component the sharing arcs are constrained to connect identical subterms, the definition of an abstract sharing component requires only that sharing arcs connect nodes having the same label. We opted for the weaker condition in the case of abstract sharing components because it simplifies the primitive operations and safety proofs, while retaining enough precision for the liveness analysis. As an alternative, we could require sharing arcs to connect nodes determining subgraphs with nonempty intersection (as defined in Janssens [1990] and Janssens and Bruynooghe [1992]). An even stronger condition would also take the enclosing type graph into account in order to determine whether a term, belonging to the intersection of the sets of terms represented by two nodes connected by a sharing arc, can indeed occur at two different places in some term represented by the enclosing type graph. The weaker condition allows ares in an abstract sharing component that may not be relevant with respect to the type graph they are associated with. For an illustration of some irrelevant sharing arcs see Figure 27.

The abstract primitive operations ignore the possible presence or creation of irrelevant arcs although they can have consequences for the precision of the sharing analysis. Figure 28 illustrates, that if irrelevant arcs are not immediately detected by the operations that create them, they can cause 
(a)

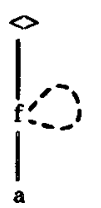

(b)

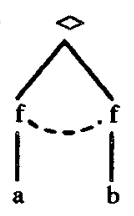

(c)

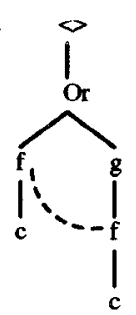

(d)

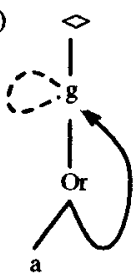

Fig. 27. Irrelevant sharing arcs-a concrete term exhibiting such sharing cannot be constructed.

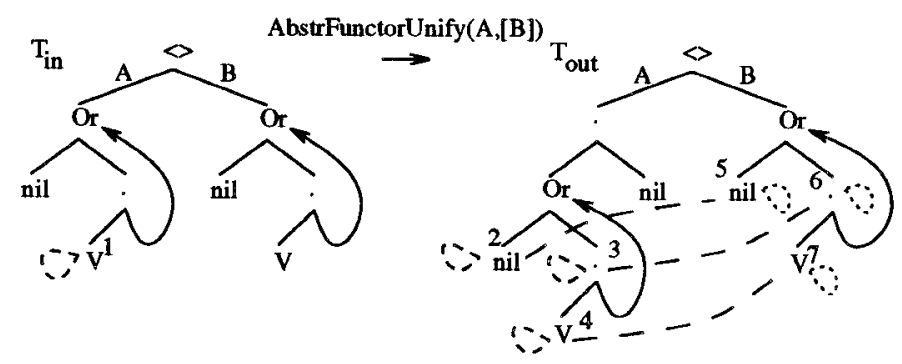

Fig. 28. Ignoring the creation of irrelevant edges causes imprecision.

imprecision in subsequent steps of the abstract interpretation, because shifting down irrelevant arcs can result in relevant ones. Suppose we have two program variables, $A$ and $B$, that are both bound to lists of free variables, and that the elements of list A possibly share with one another (type graph $T_{\text {in }}$ of Figure 28). The abstract interpretation of the unification $A=[B]$ consists of the following steps (in the figure, initial sharing arcs are drawn as short dashed lines, accumulated new arcs as long dashed lines, and arcs in the alternating closure as dotted lines).

-Expressing the sharing component for type graph $\mathrm{T}_{1 \mathrm{n}}$ (input-sharing edge $(1,1))$ in terms of type graph $\mathrm{T}_{\text {out }}$, which leads to arcs $(2,2),(3,3)$, immediately followed by an application of the shift operation w.r.t. $\mathrm{T}_{\text {out }}$, giving edge $(4,4)$.

-Constructing the arcs that correspond to places where unification possibly introduces new sharing, which leads to arcs $(2,5),(3,6)$, also immediately followed by an application of the shift operation w.r.t. $\mathrm{T}_{\text {out }}$, giving edge $(4,7)$.

- Computing the alternating closure of the sets obtained in the previous two steps, which gives the reflexive arcs in the nodes 5,6 , and 7 .

However, because the program variable $A$ is known to be a single-element list in the case of a successful unification, the input-sharing arc $(1,1)$ is in fact converted into the irrelevant arcs $(2,2)$ and $(3,3)$. If these arcs are not removed at this intermediate stage, the subsequent shift operation introduces the relevant but redundant edge $(4,4)$, and the final sharing environment is 
somewhat imprecise. In general, when as a result of unification, some Or-alternatives or back-arcs of an input type graph $\mathrm{T}_{\text {in }}$ do not have corresponding arcs in the output type graph $\mathrm{T}_{\text {out }}$, the converted version of relevant sharing arcs for $T_{\mathrm{in}}$ may be irrelevant for $\mathrm{T}_{\text {out }}$. In other cases, irrelevant reflexive arcs can also be introduced due to the conservative closure operations.

Other techniques exist to further improve the precision of the analysis, e.g., the larger the type graphs, the more precise sharing information can be. However, as one can expect, the execution time for the abstract interpretation of a program grows with the size of the type graphs used. The size (i.e., the number of nodes) of the type graphs, as derived by the abstract interpretation of a program, depends on the depth restriction for the function symbols that occur in the recursive type graph branches. The type graph operations for abstract interpretation fold the type graphs at places where the depth bound is violated. The folding of type graphs corresponds in fact to a widening operation used for convergence acceleration as discussed by Cousot [1981]. In general, an appropriate depth bound might be chosen, dynamically based on the source code of the program. We expect the optimal depth restriction for a functor occurring in a recursive branch of a type graph to be one larger than the maximal depth of the terms in selection operations occurring in the source code and involving that functor. In the prototype analyzer, the depth bound was filled in by hand. Because the test programs used were in normal form (all terms have maximal depth one), depth bound two was sufficient to get good precision; depth bound one was not. For instance, consider Figure 14 in Section 5.2. If depth bound one were used instead, the output type graph for the program variable $X_{1}$ would have been folded, and the top list cell would be indistinguishable from the other list cells. The analysis would not detect the principal list cell to become garbage after the selection.

As mentioned in Section 5.3, comparing input and output type graphs of an operation and using groundness information to remove sharing arcs that cannot possibly be introduced by the corresponding concrete operation might turn out to be another useful optimization technique. For an illustration, see Figure 29. In the output type graph shown on the right, the old sharing is represented by dashed arcs and new sharing by dotted arcs. An alternating sequence is created between two subterms that were already ground and not sharing before the unification. The current live-structure analysis yields an imprecise result in this case. A similar idea for optimization was used in Burton et al. [1992] but for a simpler abstract domain.

Also the alternating closure could be further enhanced to improve the precision in some cases. Recall that the abstract sharing component represents possible sharing and that the relation is not transitive, while each of the concrete sharing components it represents are transitive. The alternating-closure operation is very conservative in that it does not perform a case analysis of the sharings that can coexist, e.g., transitive subrelations. For instance, if there are old sharing arcs between nodes 1 and 2 , and between 2 and 3 , but not between 1 and 3, this means that the arcs 1-2 and 2-3 cannot hold in the same concrete execution state, and therefore should not be used both in an alternating sequence. Alternatively, one could keep track of sets of 

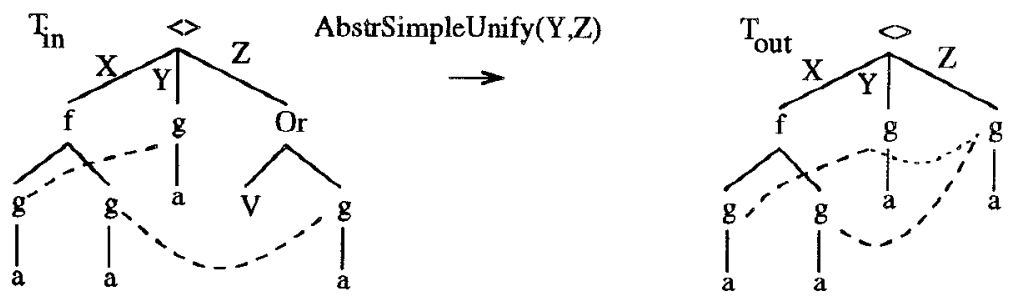

Fig. 29. Using groundness information to reduce imprecision.

sharing components, each representing a set of sharing ares that can occur in the same execution state. However, the improved precision might not be worth the extra cost.

\section{IMPLEMENTATION ISSUES AND EMPIRICAL EVALUATION}

In order to evaluate the expressiveness and precision of the domain and operations, we extended a prototype implementation written in Prolog for the abstract-interpretation framework and the type analysis by Janssens and Bruynooghe [1992]. Algorithms implementing the formal specifications of the primitive operations for the abstract domain of liveness environments were developed and incorporated into the prototype.

We applied the prototype to a set of small and well-known programs that manipulate term structures. Table I shows the analysis time in cpu seconds for some of the sample programs when executed on a Sun Microsystems SPARCstation 1, using ProLog by BIM [1990] (release 3.1.1-24 Nov. 1992). For each sample program we show the type and global liveness component of an initial call. The initial sharing components are empty, and depth bound two is used for recursive functors $(. / 2$ and $t / 3)$. The types used are defined by the following grammar rules.

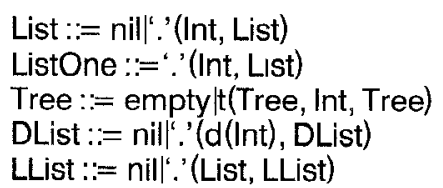

The second column shows the analysis time for the type analysis (including variable-sharing analysis), the third column for the structure-sharing analysis (including type analysis), and the fourth column for the full liveness analysis (including type and structure-sharing analysis). For small programs, the type analysis takes about 55\% of the full liveness analysis time. For larger and more complex programs, we expect the portion of sharing and liveness analysis to become larger, since after a fixpoint for the type analysis is reached, the sharing and liveness components may still differ, causing larger AND-OR graphs to be constructed and more iterations to be needed before a final fixpoint is reached. As mentioned in Section 5.2, the structuresharing analysis has to recompute the correspondence between nodes of the input and output type graphs of the primitive operations because such information is presently not exported by the type graph's abstract data type. 
Table I. Analysis Time

\begin{tabular}{l|r|r|r}
\hline CALL & type & $\begin{array}{r}\text { type }+ \\
\text { sharing }\end{array}$ & $\begin{array}{r}\text { type }+ \\
\text { sharing }+ \\
\text { liveness }\end{array}$ \\
\hline append(List,List, $\mathrm{V}^{* *}$ ) & 1.93 & 3.17 & 3.36 \\
nrev(List, $\mathrm{V}^{* *}$ ) & 4.57 & 8.15 & $\mathbf{8 . 5 5}$ \\
delete(Int,List, $\mathrm{V}^{* *}$ ) & 2.33 & 3.12 & 3.45 \\
delete(d(Int),DList, $\mathrm{V}^{* *}$ ) & 3.08 & 6.96 & 7.24 \\
setdiff(ListOne,List**, $\mathrm{V}^{* *}$ ) & 4.71 & 6.46 & 7.28 \\
insert(Int,Tree, $\mathrm{V}^{* *}$ ) & 3.22 & 5.96 & 6.23 \\
quicksort(List,List, $\mathrm{V}^{* *}$ ) & 4.77 & 7.57 & $\mathbf{8 . 3 8}$ \\
queens(Int, $\mathrm{V}^{* *}$ ) & $\mathbf{8 . 6 1}$ & $\mathbf{1 3 . 8 4}$ & 18.02 \\
matrixmult(LList,LList, $\mathrm{V}^{* *}$ ) & 10.38 & 18.17 & 20.60 \\
\hline
\end{tabular}

Because the liveness analysis uses the same correspondence relation, it explains the small time difference between structure sharing and liveness analysis. This recomputation could be avoided if liveness, sharing, and type analysis are integrated together, for instance, by using as basis a type graph unification algorithm as presented by Bruynooghe and Winsborough [1992], and the resulting analysis would be much more efficient.

Currently, the analysis works with sharing and liveness components that are closed w.r.t. the shift operation, and the depth bound for type graphs is filled in by hand. The three layers of the analysis, i.e., type, sharing, and liveness analysis, are performed simultaneously. Executing them successively might be more space efficient, as the space used on the various Prolog stacks during one layer of the analysis can be reclaimed for the next layer. It is an open question whether separating the fixpoint computation for type inferencing from the fixpoint computation of sharing, resp., liveness analysis, reduces the total analysis time. However, if the analysis is performed in separate phases, an interface between the layers has to be designed that allows us to pass on the full AND-OR graph annotated with the abstract substitutions. In order that the analysis of large programs be practical, the issues and tradeoffs involved need to be investigated.

Programs that are interesting for compile-time garbage collection are programs transforming some input data structure into a similar output data structure, after which the input data structure is no longer needed. For queries such as append(List, List, $\mathrm{V}^{* *}$ ), nrev(List, $\mathrm{V}^{* *}$ ), and quicksort(List, List, $\mathrm{V}^{* *}$ ) our prototype analyzer recognizes that the data structures being consumed are turned into garbage and that the programs offer the opportunity to reuse the garbage cells. It is possible to generate code such that the programs work in-place. The only heap memory required is that occupied by the input data structures. Note that the nrev $/ 2$ program, although it is much smaller than the quicksort $/ 3$ program, ${ }^{5}$ takes pretty much the same time to analyze. The reason is that the analysis of nrev(List, $\left.\mathrm{V}^{* *}\right)$ entails analysis of four different abstract calls of the append $/ 3$ predicate before a fixpoint is

\footnotetext{
${ }^{5}$ The second argument of the quicksort predicate is an accumulating parameter; the first is the input list of integers to be sorted; the third is the output argument.
}

ACM Transactions on Programming Languages and Systems, Vol. 16, No. 2, March 1994. 


\begin{tabular}{cc}
\hline setdiff $(X, Y, Z):-$ & delete $(P, X, Y):-$ \\
$X=n i l, Y=Z$ & $X=n i l, Y=n i l$. \\
setdiff $(X, Y, Z):-$ & delete $(P, X, Y):-$ \\
$X=[U \mid W]$, & $X=[P \mid P P], !$, \\
delete $(U, Y, I Y)$, & $\operatorname{delete}(P, P P, Y)$. \\
setdiff $(Y, I Y, Z)$. & delete $(P, X, Y):-$ \\
& $X=[Q \mid P P]$, \\
& $Y=[Q \mid Q Q]$, \\
& $\operatorname{delete}(P, P P, Q Q)$.
\end{tabular}

Fig. 3u. Set arfterence.

reached, namely

$$
\begin{array}{ll}
\text { append(nil, } & \left.[\text { Int }], \mathrm{V}^{* *}\right), \\
\text { append(nil|[Int], } & \left.[\text { Int }], \mathrm{V}^{* *}\right), \\
\begin{array}{l}
\text { append(nil|[Int]|[Int, Int }], \\
\text { append(List, }
\end{array} & \left.[\text { Int }], \mathrm{V}^{* *}\right), \text { and } \\
& \left.[\text { Int }], \mathrm{V}^{* *}\right),
\end{array}
$$

while the analysis of quicksort(List, List, $\mathrm{V}^{* *}$ ) only entails analysis of the call partition(Int, List, $\left.\mathrm{V}^{* *}, \mathrm{~V}^{* *}\right)$.

For queries such as delete(Int, List, $\mathrm{V}^{* *}$ ) (see Figure 30), more garbage cells are created (and detected) than can actually be reused within the clauses themselves. In the third clause, the selection operation $X=[Q \mid P P]$ is immediately followed by the construction operation $Y=[Q \mid Q Q]$. In the second clause there is only a selection operation. The creation of the garbage cell is detected by the prototype analyzer, but there is no opportunity for local reuse. Run-time garbage collection is still needed to discard these garbage cells, unless some techniques for nonlocal reuse are developed. The same result holds for other list types, e.g., for the call specification delete(d(Int), DList, $\left.\mathrm{V}^{* *}\right)$. In this case, the success pattern, delete(d(Int), DList, DList), indicates that the lists of the second and third argument share their elements (which have type $d(I n t)$ ). The amount of storage that is reused depends on the specific call of the predicates, because the concrete input arguments determine the execution frequency of the different clauses for the predicate.

Some garbage cells may pass undetected, e.g., for a call specification $\mathrm{p}($ List, List) and a program rule

$$
p(X, Y) \text { :- setdiff(X, } Y, Z) \text {, use }(Y) \text {, use }(Z) \text {. }
$$

the predicate setdiff $/ 3$ is called as setdiff(List, List**, $\left.\mathrm{V}^{* *}\right)$. Due to the first clause of the predicate setdiff/3, possible sharing between the output lists for $Y$ and $Z$ is derived, such that for the call use( $Y$ ), the list passed on by $Y$ is derived to be live. In concrete cases where $X$ is not nil on entry of the rule for $\mathrm{p} / 2$ however, the list cells of the list that $\mathrm{Y}$ is bound to are in fact not locally live when use $(Y)$ is called (copies of the cells have been made to construct the value of Z). For the call specification $p$ (ListOne, List) (where type ListOne means the list has at least one element), the first clause of the setdiff $/ 3$ predicate is not applicable at first, such that no sharing between the output lists for $Y$ and $Z$ is derived. Note that the call setdiff(ListOne, List**, $\mathrm{V}^{* *}$ ) entails a different recursive call setdiff(List, List, $\left.\mathrm{V}^{* *}\right)$. The corresponding call 
specifications for the delete $/ 3$ predicate in the second clause are delete(Int, List**, $\mathrm{V}^{* *}$ ) and delete(Int, List, $\mathrm{V}^{* *}$ ) respectively. The analysis of the former will (correctly) prohibit local reuse in the third clause of the delete $/ 3$ predicate.

We have also applied our prototype to analyzing tree-manipulating programs, such as buildtree $/ 2$, which transforms a list into a sorted binary tree. The analysis detects when list cells become garbage; however these cells cannot be reused locally. Analyzing buildtree $/ 2$ entails also analysis of insert $/ 3$ (program see Figure 21, Section 5.3), which inserts one element into a sorted binary tree. Our prototype's analysis of insert $/ 3$ indicates that the tree can be modified in-place. This will result in the same memory usage as one obtains by using open-ended trees.

If the query specifications for the studied programs allow sharing at the element level of the input lists or trees, essentially the same results are obtained. For example, when reversing a list of free variables that share (e.g., nrev( $(X, Y, X, Y, Z]$, Out)), the reverse procedure can still work in-place, because only the second argument field of the list cells has to be overwritten while constructing the reversed list, and these are dead, nonvariable, and not shared prior to the selection operations. However, if sharing is allowed between different input lists (trees) at the list-cell (tree-cell) level, it is generally unsafe to reuse the cells. The safe approximation achieved by our analyzer typically allows no cells to be reused in those cases.

To assess the degree of precision that our analysis can reach on larger programs, more work is needed on the efficiency of the algorithms and representation of liveness environments.

The use that a compiler can make of the liveness information that is derived varies with the sequencing of construction and selection operations in the source programs. In some cases a reordering of subgoals may be desirable. For instance, when the append $/ 3$ program is used to split a list into two sublists (call specification append $\left(\mathrm{V}^{* *}, \mathrm{~V}^{* *}, \mathrm{List}\right.$ ), program see Figure 12, Section 5.2), the compiler has to reorder the unification operations in order to benefit from the liveness information, because the construction precedes the selection operation.

To allow nonlocal reuse, an integration of the liveness analysis and the storage allocation algorithm of the compiler are needed in order to have the liveness environments reflect what garbage cells are back in use. Presently, the analyzer assumes that no garbage cells are reused. Consequently, the dead cells detected at program points following some procedure call that is not a selection operation itself are only true garbage cells if they were not already reused inside the procedure (otherwise they may be dangling references).

\section{RELATED WORK}

\section{Imperative Languages}

The problem of structure sharing and liveness is significantly different in the context of imperative languages, which have explicit construct and select 
operations instead of general term matching. Nevertheless, solutions to related structured-data dependency problems have appeared in the literature.

Horwitz et al. [1989] describe a technique, based on an analysis method introduced by Jones and Muchnick [1981], to determine an approximation of the actual layouts of memory that can arise at each program point during execution. Their method is intended for languages that have pointer-valued variables, heap-allocated storage, and a destructive update operation. It is based on store graphs that model a collection of linked structures in memory: the nodes correspond to instances of a structure type; the directed edges represent pointers between the structures; summary nodes are used to join several nodes of the graph into a single node, thereby making it possible to represent unbounded chains of run-time objects. The alias graphs finitely represent the potential sharing of structure visible at any point in the program.

Barth [1977] studied the use of global-flow analysis to determine statically the extent of objects in an attempt to reduce the number of run-time reference count operations.

Chase et al. [1990] also look at liveness analysis for imperative languages, where full unification is not an issue. Their approach summarizes the linked data structures allocated in a heap by a storage shape graph, in which one node corresponds to possibly many nodes in the heap. As in the approach of Bruynooghe and Janssens [1988], Janssens [1990], and Janssens and Bruynooghe [1992], the authors avoid the loss of information incurred in prior contributions by imposing a $k$-depth bound beyond which the structure is collapsed to a single node [Horwitz et al. 1989; Jones and Muchnick 1981; Larus and Hilfinger 1988]. As for the type inferencing used as the first layer of our liveness analysis, they rather seek to fold the structure into something like a rational tree or tree automaton. The heuristical method they use to fold the tree consists of superimposing only structure cells that are generated by the same instruction. The approach of Janssens and Bruynooghe has been to impose a fixed bound on the depth beyond which folding is required. The approach of Chase et al. in effect enables different bounds to be used for different programs, while preserving the finite ascending-chain property of the abstract domain, needed to ensure termination. To derive information useful for compile-time garbage collection, the storage shape analysis is followed by a heap reference-counting analysis, during which the nodes of the storage shape graphs are annotated with reference counts from the lattice $\{0,1, \infty\}$ (with increment $\infty+1 \rightarrow \infty$, and decrement $\infty-1 \rightarrow \infty$ operations).

\section{Functional Programming Languages}

In functional languages, sharing is introduced through parameter passing and construction of function return values. Basically, the return values of selector functions, like car and cdr, introduce sharing at positions that are known statically. However, for functional languages using lazy evaluation, deriving liveness information requires knowledge about the order in which function applications will be reduced. 
Hudak and Bloss [1985] address the problem of efficiently implementing aggregates in functional programming systems. The aggregates considered are contiguous data structures containing only atomic data types that cannot be shared. The authors present a combination of both compilation and run-time techniques for avoiding the copying of such data structures. Hudak [1987] addresses the same problem but uses abstract interpretation to infer at various points in the source program upper bounds on possible reference counts for structures. Reference counts larger than a certain chosen bound are approximated by the special value $\infty$. On function call, the reference count of each actual parameter is increased by one less than the number of occurrences of the corresponding formal parameter in the body of the function. This approach is inadequate for logic programming because whether and where new references are introduced upon unification depends on the run-time values being unified.

Similarly, Jensen and Mogensen [1990] define a domain of contexts to count the usage of expressions in a functional language. The context semantics consists of the standard semantics instrumented with a mechanism to compute the exact number of uses of expressions. The standard semantics is then abstracted away to yield an approximating analysis. They consider an abstract domain associating counts to terms and their subterms. ABS represents an undefined context or a value not used in the remaining computation; 0 represents an atomic value; $n\left(d_{1}, d_{2}\right)$ means that the value may be structured and that the top-level cell may be used $n$ times (one time if $n=1$, many times if $n=2$ ); the substructures are used as described by $d_{1}$ and $d_{2}$.

Inoue and Torii [1991] and Inoue et al. [1988] handle the data manipulation operations of cons, car, cdr, and parameter passing, but their work is insufficient to handle general unification. The approach consists of deriving from the functional program text the set of strings of list type primitive functions (cons, car, cdr) that are possibly applied to program variables. These strings of primitive operations are translated into occurrences (expressions of the form $\{0,1\}^{*}$, where 0 selects the head and 1 the tail of a list). Overlapping (shared) cells are cells that have more than one occurrence relative to the root. However, no full sharing analysis is performed; input arguments are assumed to be nonoverlapping.

Park and Goldberg [1992] present a more general escape analysis for higher-order functional programs, the purpose of which is to determine which (parts of) arguments to a function call are returned by that call. The results of the escape analysis can be used for a sharing analysis of lists.

\section{Logic-Programming Languages}

Previous work on aliasing in logic-programming languages mainly addressed the sharing of free variables. For the application of liveness analysis, the sharing of structured terms becomes relevant also, as was discussed in Section 2.

\section{Variable Sharing}

Techniques for deriving information about shared variables in logic programs for the application of occur-check reduction can be found in Jones and 
Søndergaard [1987], Mellish [1985; 1987], Plaisted [1984], and Søndergaard [1986]. In groundness analysis [Codish et al. 1990; 1991; Cortesi et al. 1991], mode and type analysis [Debray and Warren 1986; Janssens 1990; Janssens and Bruynooghe 1992; Kluźniak 1987; Mellish 1987], and independence analysis [Jacobs and Langen 1989; Muthukumar and Hermenegildo 1989], tracing the dependencies between free variables plays a key role in preserving the soundness of the analysis.

\section{Structure Sharing and Liveness}

Vataja and Ukkonen [1984] consider the problem of garbage collection in the context of backtrack-based Prolog implementations. The key concept of their analysis is that of a temporary term, which is a structured term that is not shared at some program point with the calling environment. Intuitively, a temporary term is a term needed for the local purposes of some clause. A term is strongly temporary if it is not shared with any term of a backtrack point in the search tree for the clause activation. At the end of the clause activation, the storage space reserved for the strongly temporary terms can be reclaimed. This concept is rather strong and prevents the introduction of destructive assignments in many cases. Also, it assumes some determinacy analysis. On the other hand, the trailing mechanism does not have to be adapted, as is the case in our proposal. Although their approach includes a full sharing analysis (based on depth- $k$ abstractions and color variables), it is described only informally, and no correctness proofs are provided.

An approach to liveness analysis for the significantly restricted case of so-called Ground Prolog has been developed by Kluźniak [1988]. Ground Prolog is a restricted form of pure Prolog in the sense that the query must be known; mode declarations must be given for each predicate; and the groundness restriction is imposed such that whenever a call is executed, (1) its input parameters must be ground and (2) the output parameters must be free. As a consequence, general unification need not be handled.

\section{CONCLUSION}

In the present article, we reviewed an abstract domain and primitive operations to compute structure sharing and liveness information for logic programs at both the concrete and abstract levels. Formal specifications and safety proofs have been worked out in Mulkers [1991], Mulkers et al. [1990b], and Mulkers et al. [1993]. The contribution of the present work is a thorough discussion of the implementations for which the analysis is suited. We also pointed out some design alternatives that might further improve the accuracy of the live-structure information. We empirically investigated the precision of the present analysis and demonstrated that it is sufficient to recognize opportunities for compile-time garbage collection in a variety of sample programs.

\section{REFERENCES}

BARTH, J. M. 1977. Shifting garbage collection overhead to compile time. Commun. ACM 20, 7 (July), 513-518.

BIM 1990. ProLog by BIM-3.0.-Reference Manual. B-3078, BIM, Everberg, Belgium. 
Boizumault, P. 1988. PROLOG L'Implantation Masson, Paris. In French.

BRUYNOOGHE, M. 1991 A practical framework for the abstract interpretation of logic programs. J. Logic Program. 10, 2 (Feb.), 91-124.

Bruynooghe, M., AND Janssens, G. 1988. An instance of abstract interpretation integrating type and mode inferencing. In Proceedings of the 5th International Conference and Sympostum on Logic Programming. MIT Press, Cambridge, Mass, 669-683.

Bruynooghe, M., And Winsborough, W. 1992. Type graph unification. Rep. CW160, Dept. of Computer Science, Katholieke Universiteit Leuven, Belgium.

Bruynooghe, M., Janssens, G., Callebaut, A., and Demoen, B. 1987. Abstract interpretation: Towards the global optimization of Prolog programs. In Proceedings of the 4th Symposium on Logic Programming. IEEE Computer Society Press, Los Alamitos, Calif, 192-204.

Burton, B., Gudjonsson, G., and Winsborough, W. 1992. An algorithm for computing alternating closure. Tech. Rep. CS-92-15, Dept. of Computer Science, The Pennsylvania State Univ University Park, Pa.

Chase, D. R., WEGMan, M., AND ZADECK, F. K. 1990, Analysis of pointers and structures. In Proceedings of ACM SIGPLAN '90 Conference on Programming Language Design and Implementation. SIGPLAN Not. 25, 6, 296-310.

Codish, M., Dams, D., AND YARDeni, E. 1991. Derivation and safety of an abstract unification algorithm for groundness and aliasing analysis. In Proceedings of the 8th International Conference on Logic Programming. MIT Press, Cambridge, Mass., 79-93.

Codish, M., DAms, D., AND YARDENI, E. 1990. Abstract unification and a bottom-up analysis to detect aliasing in logic programs. Tech. Rep. CS90-10, Dept. of Computer Science. Weizmann Institute of Science, Israel.

Colmerauer, A. 1982. Prolog and infinite trees. In Loglc Programming. Academic Press, New York, 231-251.

Contesi, A., AND Filé, G. 1991 Abstract interpretation of Prolog. The treatment of the built-ins. Rapporto Interno 11, Dept, of Mathematics, Univ. of Padoua.

Cortesi, A., Filê, G., And Winsborough, W. 1991. Prop revisited: Propositional formula as abstract domain for groundness analysis. In Proceedings of the 6th Annual IEEE Symposium on Logic in Computer Science. IEEE Computer Society Press, Los Alamitos, Calif., 322-327.

Cousot, P. 1981. Semantic foundations of program analysis. In Program Flow Analysis: Theory and Applications. Prentice-Hall, Englewood Cliffs, N.J., 303-342

Cousot, P., AND Cousot, R. 1992. Comparing the Galois connection and widening/narrowing approaches to abstract interpretation. In Proceedings of the International Workshop PLILP '92. Lecture Notes in Computer Science, vol 631. Springer-Verlag, Heidelberg, Germany, 269-295.

Cousot, P., AND Cousot, R. 1977. Abstract interpretation: A unified lattice model for static analysis of programs by construction or approximation of fixpoints In Proceedings of the 4th ACM Symposium on Principles of Programming Languages. ACM, New York, 238-252.

DEBRAY, S. K. 1992. Efficient dataflow analysis of logic programs. $J A C M 39,4$ (Oct.), 949-984.

Debray, S. K., AND WARREN, D. S. 1986a. Automatic mode inference for Prolog programs. In Proceedings of the 3rd Symposium on Logic Programming. IEEE Computer Society Press, Los Alamitos, Calif., 78-88.

DEBRAY, S. K., AND WARREN, D. S. 1986b. Detection and optimization of functional computations in Prolog. In Proceedings of the 3rd International Conference on Logic Programming. Lecture Notes in Computer Science, vol. 225. Springer-Verlag, New York, 490-504.

FosTer, I. 1989. Copy avoidance through local reuse. Tech. Rep. Preprint MCS-P99-0989, Mathematics and Computer Science Div., Argonne National Lab

Foster, I., AND Winsborough, W. 1991. Copy avoidance through compile-time analysis and local reuse. In Proceedings of the International Logic Programming Symposium MIT Press, Cambridge, Mass., 455-469.

GÈCSEG, F., AND STEInBY, M. 1984. Tree Automata. Akadémiai Kiadó.

Horwitz, S., Pfeiffer, P., AND Reps, T. 1989. Dependence analysis for pointer variables. In Proceedings of the ACM SIGPLAN '89 Conference on Programming Language Design and Implementation. SIGPLAN Not. 24, 7, 28-40.

ACM Transactions on Programming Languages and Systems, Vol 16, No. 2, March 1994. 
Hudak, P. 1987. A semantic model for reference counting and its abstraction. In Abstract Interpretation of Declarative Languages. Ellis Horwood, Chichester, 45-62.

HudaK, P., AND Bloss, A. 1985. The aggregate update problem in functional programming systems. In the 12th Annual ACM Symposium on Principles of Programming Languages. ACM, New York, 300-314.

HuET, G. 1980. Confluent reductions: Abstract properties and applications to term rewriting systems. J. ACM 27, 4, 797-821.

INOUE, K., AND TORII, K. 1991. Implementation and analysis of compile-time garbage collection. New Gen. Comput. 10, 1, 101-119.

InOUe, K., SeKI, H., AND YAGI, H. 1988. Analysis of functional programs to detect run-time garbage cells. ACM Trans. Program. Lang. Syst. 10, 4, 555-578.

JACOBS, D., AND LANGEN, A. 1989. Accurate and efficient approximation of variable aliasing in logic programs. In Proceedings of the North American Conference on Logic Programming. MIT Press, Cambridge, Mass., 154-165.

JANSSENS, G. 1990. Deriving run time properties of logic programs by means of abstract interpretation. Ph.D. thesis, Dept. of Computer Science, Katholieke Univ. Leuven, Belgium.

JANssens, G., AND BRUYNoOGHe, M. 1992. Deriving descriptions of possible values of program variables by means of abstract interpretation. J. Logic Program. 13, 2 and 3 (July), 205-258.

Janssens, G., Demoen, B., ANd Whllems, Y. 1987. Execution mechanism for Prolog. Rep. CW53, Dept. of Computer Science, Katholieke Univ. Leuven, Belgium.

Jensen, T. P., AND Morgensen, T. Æ. 1990. A backwards analysis for compile-time garbage collection. In ESOP '90 Proceedings of the 3rd European Symposium on Programming. Lecture Notes in Computer Science, vol. 432. Springer-Verlag, New York, 227-239.

Jones, N. D., AND MUCHNICK, S. S. 1981. Flow analysis and optimization of LISP-like structures. In Program Flow Analysis: Theory and Applications. Prentice-Hall, Englewood Cliffs, N.J., 102-131.

Jones, N. D., AND SøNDergaard, H. 1987. A semantic-based framework for the abstract interpretation of Prolog. In Abstract Interpretation of Declarative Languages. Ellis Horwood, Chichester, 123-142.

KLUŹNIAK, F. 1988. Compile-time garbage collection for ground Prolog. In Proceedings of the 5th International Conference and Symposium on Logic Programming. MIT Press, Cambridge, Mass., 1490-1505.

KLUŹNLAK, F. 1987. Type synthesis for ground Prolog. In Proceedings of the 4th International Conference on Logic Programming. MIT Press, Cambridge, Mass., 788-816.

LARUS, J. R., AND HILFINGER, P. N. 1988. Detecting conflicts between structure accesses. In Proceedings of the ACM SIGPLAN '88 Conference on Programming Language Design and Implementation. SIGPLAN Not. 23, 7, 21-34.

LASSEz, J.-L., MAHER, M. J., AND MARRIOTT, K. 1988. Unification revisited. In Foundations of Deductive Databases and Logic Programming. Morgan Kaufmann, Los Altos, Calif., 587-625.

Mariën, A., Janssens, G., Mulkers, A., AND Bruynooghe, M. 1989. The impact of abstract interpretation: An experiment in code generation. In Proceedings of the 6th International Conference on Logıc Programming. MIT Press, Cambridge, Mass., 33-47.

Marriott, K., AND SøndergaARd, H. 1989. Semantics-based dataflow analysis of logic programs. In Information Processing 89. North-Holland, New York, 601-606.

MELLISH, C. S. 1987. Abstract interpretation of Prolog programs. In Abstract Interpretation of Declarative Languages. Ellis Horwood, Chichester, 181-198.

MELLish, C. S. 1985. Some global optimizations for a Prolog compiler. J. Logic Program. 2, $43-66$.

MulkERS, A. 1991. Deriving live data structures in logic programs by means of abstract interpretation. Ph.D. thesis, Dept. of Computer Science, Katholieke Univ. Leuven, Belgium. To appear in Lecture Notes in Computer Sctence, vol. 675.

Mulkers, A., Winsborough, W., AND Bruynooghe, M. 1993. A live-structure data-flow analysis for Prolog: Theory. Rep. CW167, Dept. of Computer Science, Katholieke Univ. Leuven, Belgium.

Mulkers, A., Winsborough, W., And Bruynooghe, M. 1992. Static analysis of logic programs to detect run-time garbage cells. In Proceedings of the International Conference on Computer 
Systems and Software Engineering. IEEE Computer Society Press, Los Alamitos, Calif., 526-531.

Mulkers, A., Winsborough, W., And BruYnooghe, M. 1990a. Analysis of shared data structures for compile-time garbage collection in logic programs. In Proceedings of the 7 th International Conference on Logic Programming. MIT Press, Cambridge, Mass., 747-762.

Mulkers, A., Winsborough, W., And Bruynooghe, M. 1990b. Analysis of shared data structures for compile-time garbage collection in logic programs (extended version). Rep. CW117, Dept. of Computer Science, Katholieke Univ. Leuven, Belgium.

Muthukumar, K., And Hermenegildo, M. 1989. Determination of variable dependence information through abstract interpretation. In Proceedings of the North American Conference on Logic Programming. MIT Press, Cambridge, Mass., 166-185.

Nilsson, U. 1990. Systematic semantic approximations of logic programs. In Proceedings of the International Workshop on Programming Language Implementation and Logic Programming. Lecture Notes in Computer Science, vol. 456. Springer-Verlag, New York, 293-306.

Park, Y. G., AND Goldberg, B. 1992. Escape analysis on lists. In Proceedlngs of ACM SIGPLAN '92 Conference on Programming Language Design and Implementation. SIGPLAN Not. 27, 7, 116-127.

Plaisted, D. A. 1984. The occur-check problem in Prolog. J. New Gen. Comput. 2, 4, 309-322.

SøNDERGAARD, H. 1986. An application of abstract interpretation of logic programs: Occur check reduction. In ESOP '86 Proceedings of the European Symposium on Programming Lecture Notes in Computer Science, vol. 213. Springer-Verlag, New York, 327-338.

TARSKI, A. 1955. A lattice-theoretical fixpoint theorem and its applications. Paciftc J. Math. 5, 285-309.

TAYlor, A. 1991. High performance Prolog implementation. Ph.D. thesis, Univ. of Sydney, Australia.

TAYLOR, A. 1990. LIPS on a MIPS, results from a Prolog compiler for a RISC. In Proceedings of the 7th International Conference on Logic Programming. MIT Press, Cambridge, Mass., $174-185$.

TAYLOR, A. 1989. Removal of dereferencing and trailing in Prolog compilation. In Proceedlngs of the 6th International Conference on Logic Programming. MIT Press, Cambridge, Mass., $48-60$.

Van Caneghem, M. 1986. L'Anatomie de Prolog. InterEditions. In French.

VAN RoY, P. L. 1990. Can logic programming execute as fast as imperative programming. $\mathrm{Ph.D}$. thesis, Univ. of California, Berkeley, Calif.

Van Roy, P. L., ANd Despain, A. M. 1990. The benefits of global dataflow analysis for an optimizing Prolog compiler. In Proceedings of the 1990 North Amertcan Conference on Logic Programming. MTT Press, Cambridge, Mass., 501-515.

Vata.jA, P., AND UkKonen, E. 1984, Finding temporary terms in Prolog programs. In Proceedings of the International Conference on Fifth Generation Computer Systems Ohmsha, LTD and North-Holland, Tokyo/Amsterdam, 275-282.

WarRen, D. H. D. 1983. An abstract Prolog instruction set. Tech. Rep., SRI International, Artificial Intelligence Center, Menlo Park, Calif.

Received May 1992; revised February 1993; accepted May 1993 